\title{
MOSFET DETECTORS IN QUALITY ASSURANCE OF TOMOTHERAPY TREATMENTS \\ by
}

\author{
Amanda Cherpak \\ B.Sc. in Physics (2005) \\ A thesis submitted to the \\ Faculty of Graduate Studies and Research \\ in partial fulfillment of the requirements \\ for the degree of \\ Master of Science \\ Ottawa-Carleton Institute for Physics \\ Department of Physics, Carleton University \\ Ottawa, Ontario, Canada \\ July, 2007 \\ (C) Copyright 2007, Amanda Cherpak
}




$\begin{array}{ll}\begin{array}{l}\text { Library and } \\ \text { Archives Canada }\end{array} & \begin{array}{l}\text { Bibliothèque et } \\ \text { Archives Canada }\end{array} \\ \begin{array}{l}\text { Published Heritage } \\ \text { Branch }\end{array} & \begin{array}{l}\text { Direction du } \\ \text { Patrimoine de l'édition }\end{array} \\ \begin{array}{l}\text { 395 Wellington Street } \\ \text { Ottawa ON K1A 0N4 }\end{array} & \begin{array}{l}\text { 395, rue Wellington } \\ \text { Ottana ON K1A ON4 } \\ \text { Canada Oa }\end{array}\end{array}$

Your file Votre référence ISBN: 978-0-494-33694-6 Ourfile Notre référence ISBN: 978-0-494-33694-6

NOTICE:

The author has granted a nonexclusive license allowing Library and Archives Canada to reproduce, publish, archive, preserve, conserve, communicate to the public by telecommunication or on the Internet, loan, distribute and sell theses worldwide, for commercial or noncommercial purposes, in microform, paper, electronic and/or any other formats.

The author retains copyright ownership and moral rights in this thesis. Neither the thesis nor substantial extracts from it may be printed or otherwise reproduced without the author's permission.
AVIS:

L'auteur a accordé une licence non exclusive permettant à la Bibliothèque et Archives Canada de reproduire, publier, archiver, sauvegarder, conserver, transmettre au public par télécommunication ou par l'Internet, prêter, distribuer et vendre des thèses partout dans le monde, à des fins commerciales ou autres, sur support microforme, papier, électronique et/ou autres formats.

L'auteur conserve la propriété du droit d'auteur et des droits moraux qui protège cette thèse. $\mathrm{Ni}$ la thèse ni des extraits substantiels de celle-ci ne doivent être imprimés ou autrement reproduits sans son autorisation.
In compliance with the Canadian

Privacy Act some supporting forms may have been removed from this thesis.

While these forms may be included in the document page count, their removal does not represent any loss of content from the thesis.
Conformément à la loi canadienne sur la protection de la vie privée, quelques formulaires secondaires ont été enlevés de cette thèse.

Bien que ces formulaires aient inclus dans la pagination, il n'y aura aucun contenu manquant. 


\section{Abstract}

In vivo dosimetry is a useful tool which can help identify and correct errors in treatment planning, patient set-up, and treatment delivery. The purpose of this work was to characterize metal oxide semiconductor field-effect transistors (MOSFETs) in a $6 \mathrm{MV}$ conventional linac and to investigate their potential as in vivo dosimeters for quality assurance of radiotherapy treatments with a Tomotherapy Hi-Art Unit. High sensitivity and standard sensitivity MOSFETs were first calibrated and then tested for reproducibility, field size dependence, and accuracy of measuring surface dose in a $6 \mathrm{MV}$ beam as well as in a Tomotherapy Hi-Art unit. "In vivo" measurements were performed on both a RANDO phantom and several head and neck cancer patients treated with Tomotherapy and compared to TLD measurements and treatment plan doses to evaluate the performance of MOSFETs in a high gradient radiation field. 


\section{Acknowledgements}

I would like to express my sincere thanks to my supervisor, Joanna Cygler, for all of her guidance, teaching and support which has made this work possible. I have learned a great deal from her over the past two years, and physics is only the beginning. I would like to thank Ryan Studinski, who spent countless hours helping me with experiments and who l'm sure helped me avoid many expensive mistakes. I would also like to thank Miller MacPherson and Lee Gerig for their insightful discussions and ideas regarding this work. My deepest thanks go to Kenji, Essi, Maria, Jared, Tara and Leah for making long days in the lab seem a little less long, for always keeping me laughing, and for making these past two years so much fun. I have sincerely enjoyed working with every one of them. My family here in Ottawa; Deirdre, Paul, Megan, Liam and Brennan have always been there for me since I first arrived in this city and have shown me love and kindness that I will never forget. 
I cannot express how grateful I am to my parents as well as my two sisters, Mary Rachelle and Lindsay, who have always been only a phone call away, willing to help me through my frustrations and to celebrate my successes. Finally, I would like to dedicate this work to my dad, for supporting me every step of the way. Among many other things, he has taught me to value education and to never stop learning. I am sure this thesis would not have come to be without his constant love and guidance. 


\section{Contents}

Abstract iii

Acknowledgements iv

1. Introduction 1

2. In vivo dosimeters 5

2.1 Metal oxide semiconductor field-effect transistors.................... 5

2.1.1 Structure of MOSFETs........................................... 5

2.1.2 Principle of MOSFETs........................................... 7

2.1.3 Operation of MOSFET dosimetry systems..................... 9

2.1.4 Types of MOSFETs available................................... 11

2.1.5 Characteristics of microMOSFETs........................... 14

2.2 Thermoluminescence dosimeters................................. 19

2.2.1 Physics of TLDs.............................................. 19

2.2.2 Advantages and Disadvantages of TLDs..................... 21

2.3 Diodes.............................................................. 22

2.3.1 Physics of Diodes........................................... 22

2.3.2 Characteristics of Diodes................................... 22 
2.4 Optically stimulated luminescence dosimeters........................ 23

3. Intensity modulated radiation therapy and Tomotherapy 25

3.1 Intensity Modulated Radiation Therapy.............................. 25

3.2 Tomotherapy....................................................... 27

4. Characterization of MOSFETs used in this study 29

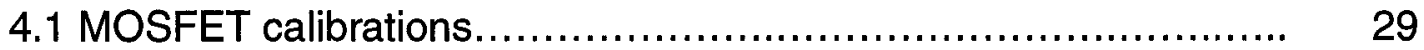

4.1.1 Calibrations on a 6 MV linac.................................... $\quad 29$

4.1.2 Calibrations in a Cobalt-60 beam............................. 32

4.1.3 Calibration factor dependence on field size and accumulated dose.................................................................... 32

4.1.4 Calibrations on a Tomotherapy Unit.......................... 33

4.1.5 Results....................................................... 35

4.2 MOSFET Surface Dose Measurements............................... 41

4.2.1 Procedures .................................................. 41

4.2.2 Results........................................................ 43

5. In vivo Measurements 49

5.1 "In vivo" Measurements with Anthropomorphic Rando Phantom..... 49

5.1.1 Treatment Preparation........................................ 49

5.1.2 Reproducibility Measurements ................................ 54

5.1.3 Evaluation of Skin as Organ at Risk......................... 57

5.1 .4 Results....................................................... 57

5.2 In vivo Measurements with Head and Neck Patients................. 64

5.2.1 Procedures...................................................... 64

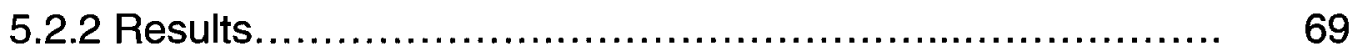

$\begin{array}{ll}\text { 6. Conclusions } & 75\end{array}$

vii 


\section{List of Tables}

4.1 MOSFET calibration factors measured in a $6 \mathrm{MV}$ beam................ 36

4.2 MOSFET calibration factors measured in a Cobalt-60 beam............ 36

5.1 List of organs at risk and target volumes contoured for treatment

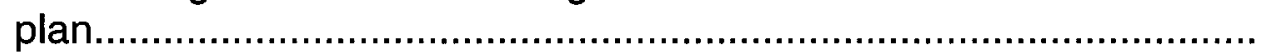

5.2 "In vivo" dose measurements and Tomotherapy treatment plan dose values for RANDO phantom.....................................

5.3 MOSFET measured doses for treatment of RANDO phantom with Plan 1, with no skin sparing, and Plan 2, with skin sparing............ 62

5.4 In vivo dose measurements and Tomotherapy treatment plan dose values for 'Patient $E$ ' treated on Tomotherapy. 


\section{List of Figures}

2.1 MOSFET structure. Diagram adapted from M Soubra, J Cygler, and G Mackay, "Evaluation of dual bias dual metal oxide-silicon semiconductor field effect transistor detector as radiation dosimeter," Med Phys. 1994; 21, 567-572.......................... 6

2.2 Change in threshold voltage with exposure to radiation..................... 8

2.3 a. Bubble-up and b. bubble-down MOSFET orientations with respect to the beam. Diagram is not to scale.....................................

2.4 TN-RD-20 Standard Bias Supply and DPU-411 Type II Thermal Printer

2.5 Effect of accumulated dose on the relative response of a standard MOSFET sensor. Graph from R Ramaseshan, $S$ Russell, and $P$ O'Brien, "Clinical dosimetry using MOSFETs" Int. J. Radiat. Onc. Biol. Phys. 1997; 37, 959-964

2.6 The change in MOSFET signal with consecutive readings plotted versus time after initial reading, a phenomenon known as "the creep-up effect". Graph from R Ramaseshan, S Russell, and P O'Brien, "Clinical dosimetry using MOSFETs" Int J Radiat Onc Biol.

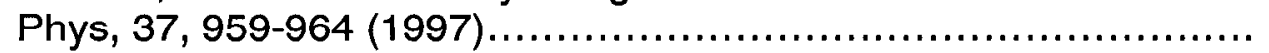

2.7 A typical thermogram (glow curve) of a LiF:Mg,Ti TLD chip as measured by a Harshaw TLD reader.

3.1 Diagram showing the geometry for a. IMRT and b. helical 28 Tomotherapy treatment delivery. In each case, the beam (shown in blue) is intensity modulated, however, for IMRT, treatment is 
delivered from only a few angles, while with tomotherapy, the dose is delivered from a full $360^{\circ}$ as the patient (represented by yellow circle) moves through the bore of the machine.

4.1 Experimental set-up for MOSFET calibrations in a conventional linac beam

4.2 Experimental set-up for MOSFET calibrations using a Tomotherapy hi-art unit beam

4.3 Relative MOSFET calibration factors for $10 \times 10 \mathrm{~cm}^{2}, 15 \times 15 \mathrm{~cm}^{2}$, $20 \times 20 \mathrm{~cm}^{2}$ and $25 \times 25 \mathrm{~cm}^{2}$ field sizes for standard sensitivity and high sensitivity MOSFETs

4.4 Average calibration factors for all MOSFETs tested normalized to the individual detector response at $10 \times 10 \mathrm{~cm}^{2}$, for field sizes $10 \times 10$ $\mathrm{cm}^{2}-25 \times 25 \mathrm{~cm}^{2}$ for standard sensitivity and $4 \times 4 \mathrm{~cm}^{2}-25 \times 25 \mathrm{~cm}^{2}$ for high sensitivity....

4.5 Relative MOSFET calibration factors for a. four standard sensitivity MOSFETs and $b$. four high sensitivity MOSFETs measured at various points throughout lifetime of each detector

4.6 Percent-depth dose curve as measured by a Markus ion chamber with a linear fit of the build-up region.

4.7 Comparison of a. standard sensitivity MOSFET and b. high sensitivity MOSFET surface dose readings and the expected surface dose, as calculated from ion chamber readings................

4.8 Percent depth dose curve measured with a high sensitivity MOSFET. Data is presented both with and without shift of water equivalent depth.

4.9 Comparison of percent depth dose curves as measured by a standard sensitivity MOSFET, a high sensitivity MOSFET and a Markus ion chamber.

5.1 Anthropomorphic RANDO phantom and S-frame immobilization mask, with points of interest marked with bb's.

5.2 Screen capture of the treatment plan showing contours of the spinal cord, target, and skin sparing structure

5.3 Tomotherapy image registration showing the daily MVCT (grey image) and the original kVCT used for treatment planning (yellow image) a. before and b. after shifts 
5.4 Screen capture of the treatment plan showing the position of the bb and the Hounsfield unit and the total dose assigned to the voxel.....

5.5 MOSFET dose measurements taken on the RANDO phantom at five points of interest. The first three measurements at position 2 are absent due to a malfunction of the MOSFET during the experiment. The uncertainty shown is from the standard deviation of the original calibration factors

5.6 Dose-volume histograms generated from a. Plan 1, with no effort to spare the skin OAR and b. Plan 2, with sparing of the skin.

5.7 Isodose curves generated from the treatment plan for 'Patient $A$ '. The prescribed target volume, located to the right of the nose, is shown as well as areas of dose that follow the legend on the left. The red curve, for example, outlines a region that receives at least $50.4 \%$ of the prescribed dose.

5.8 The immobilization mask for 'Patient A' with MOSFETs attached to the five points of interest

5.9 MOSFET dose measurements taken on 'Patient E' at five points of interest. Measurements were taken on eight different days, and readings that were compromised due to movement of the detector during treatment have been omitted. The uncertainty shown is from the standard deviation of the original calibration factors.

5.10 A profile of Hounsfield units from a KVCT of cylindrical solid water phantom with a lung insert, as assigned by the Tomotherapy treatment planning software

5.11 A dose profile of a beam entering tissue a. perpendicular to the surface and $b$. on an angle. The depth of maximum dose, $d_{\max }$, can be brought closer to the surface by using beams entering on angle, as seen with $d_{\max 2}$, a method commonly used in head and neck treatment plans. 


\section{Chapter 1}

\section{Introduction}

Radiation therapy has been used to treat cancer for over one hundred years, beginning only a year after the discovery of the x-ray in 1895 [1]. The following century has been filled with important advancements in the production and delivery of radiation, most importantly the use of ${ }^{60} \mathrm{Co}$ as a source material and the invention of the linear accelerator [1]. The goal of any radiation treatment is to deliver a maximized uniform dose to the target, while sparing the surrounding healthy tissue as much as possible. The implementation of this idea began with the use of blocks, wedges, and multiple beam angles, which can be aided by imaging tools to better conform to the tumor volume.. In recent years, intensity modulated radiation therapy, IMRT, has been developed, which consists of a conventional linac equipped with multi-leaf collimators that open and close to either block a segment of the beam or allow it to pass, creating non uniform dose profiles [2]. Intensity modulated radiation therapy, IMRT, treatment plans can be optimized to deliver highly conformal dose to target volumes and spare specific organs at risk. The latest technology in radiation therapy is helical Tomotherapy [3]. This machine is a combination of a computed tomography scanner and linac that can deliver intensity modulated beams from a full $360^{\circ}$, providing even more control over dose distribution.

IMRT has advantages in treatment of regions of the body where critical organs at risk are in close proximity to the target volume. For this reason, it is a common treatment modality for cancers of the head and neck, where organs such as the 
parotid gland, spinal cord and eyes must be spared as much as possible. Another organ at risk with head and neck cancers is the skin. Many tumors in this region are located close to the surface, so skin sparing is limited in order to deliver an adequate dose to the target. This problem is amplified by the use of IMRT and inverse planning (described on page 26), as skin doses in IMRT tend to be higher than with conventional therapy. This is due to the use of multiple beams that enter tangentially to the skin, which are used to offset the build-up region of beams entering perpendicularly [4]. The use of these tangential beams can cause an increase in skin dose of $19 \%$ and $27 \%$, with and without an immobilization mask respectively, from a treatment in which perpendicular beams are used [5].

A higher skin dose leads to a greater possibility of skin toxicity, therefore it is important to predict and accurately monitor the dose received. This is a complex task as there are reports of inconsistencies between doses calculated by IMRT treatment planning systems and those measured through in vivo dosimetry on the surface and in build-up regions [6]. One reason for this is the partial volume effect (described in detail on page 26),, which can cause significant inaccuracies in dose calculations at interfaces between materials of different densities. Studies comparing treatment plan surface doses with radiochromic film measurements have noted overestimations of the calculated treatment plan dose of up to $18.5 \%$ (5400 cGy) with respect to the prescribed dose and errors were significant throughout the entire build-up region [6]. 
The complexities introduced by inverse treatment planning make in vivo measurements on patients during treatment with IMRT a critical part of assuring accurate dose delivery. Because of the high dose gradients present with IMRT, dosimeters with high spatial resolution are needed to accurately measure a region of homogeneous dose. Therefore, the small active volume $(<0.5 \mathrm{~mm}$ width and $25 \mu \mathrm{m}$ thickness) of MOSFETs minimizes the inaccuracy that can arise from having large dose gradients across the detector volume. Studies have shown that MOSFETs display linearity, reproducibility, and accuracy in IMRT similar to when used in a conventional beam arrangement [7].

One study compared MOSFET and Farmer-type cylindrical ion chamber measurements with calculated IMRT treatment plan doses in a solid water phantom at points beyond the dose build up region. Measurements were made in regions of high and low dose as well as in areas with high dose gradients and in more homogenous regions. The overall average agreement with the treatment plan doses was within $5 \%$ error for the MOSFET measurements and within $3 \%$ error for the ion chamber measurements. Errors in set-up and positioning of both the patient and the dosimeters can cause significant errors in IMRT measurements because of the high dose gradients. This is one of the main causes of the variation between the in vivo measurements and treatment plan doses [7]. Another study compared the results of over 800 MOSFET measurements taken in the oral cavity of patients undergoing IMRT treatment for oropharynx and nasopharynx cancers. Over $90 \%$ of the MOSFET measurements were within $\pm 5 \%$ of the calculated values and uncertainties were attributed to 
patient and MOSFET position variations between treatment fractions, known as inter-fraction variations, and patient movements during a single treatment fraction, known as intra-fraction movement [8]. Only standard sensitivity MOSFETs were used in that study, so the uncertainties could potentially be improved by the use of higher sensitivity MOSFETs. All of these studies demonstrate the suitability of MOSFETs for in vivo measurements in IMRT, as well as the potential for their use for surface dose measurements.

This thesis describes experiments and measurements completed at the Ottawa Regional Cancer Centre, Ottawa, ON, which aim to validate the need for in vivo dosimetry on head and neck cancer patients treated with helical Tomotherapy and to characterize the performance of MOSFETs under such conditions. 


\section{Chapter 2}

\section{In vivo dosimeters}

In vivo dosimetry is an important part of accurate dose delivery, as it can identify random as well as systematic errors in treatment planning and patient set-up [9]. Many different types of detectors are available for such measurements including MOSFETs, thermoluminesence dosimeters, diodes, and optically stimulated luminescence dosimeters. This chapter describes the physics behind the radiation response of each detector as well as outlines the performance characteristics when used for in vivo dosimetry.

\subsection{Metal oxide semiconductor field-effect transistors}

\subsubsection{Structure of MOSFETs}

Metal oxide-silicon semiconductor field effect transistors (MOSFETs) have been introduced as an alternative to the widely used thermoluminescent dosimeters (TLDs) for in vivo dosimetry. P-channel enhancement MOSFETs consist of a negatively doped (n-type) silicon substrate, on top of which lies the source, gate and drain terminals, as seen in Fig.2.1. The source and drain terminals are built on top of a region of positively-doped (p-type) silicon while the gate terminal is separated from the n-type silicon substrate by an insulating layer of silicondioxide. The substrate region directly below the silicon dioxide is called the channel.

The voltage applied to the gate controls the conductivity of this channel. When a negative gate voltage, $V_{G}$, is applied, free electrons are repelled from the channel, leaving an electron-depleted region, and positive charge carriers are 
attracted from the substrate into the channel, making it more conductive. When a sufficient number of positive holes builds up, the substrate in the channel changes from n-type to p-type, creating an inversion layer which connects the source, gate and drain. The voltage required to create this inversion effect and induce a conducting channel is called the threshold voltage, $V_{t h}$.

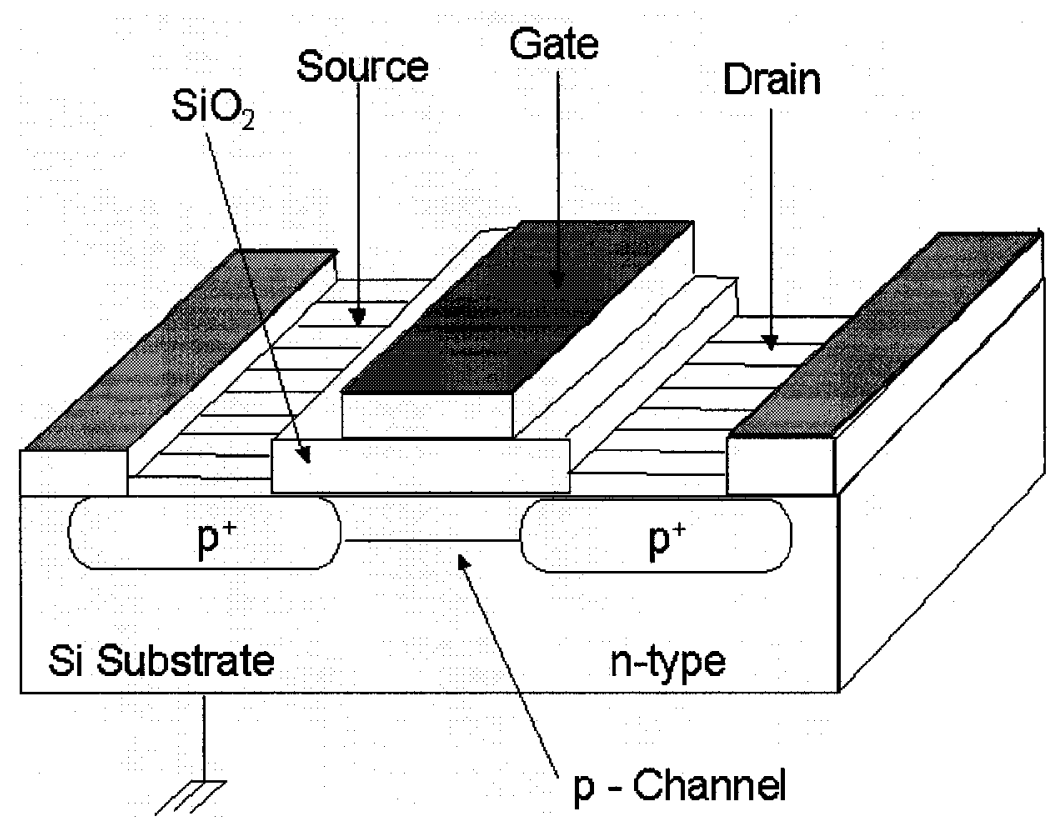

Fig.2.1 MOSFET structure. Diagram adapted from M Soubra, J Cygler, and G Mackay, "Evaluation of dual bias dual metal oxide-silicon semiconductor field effect transistor detector as radiation dosimeter," Med Phys. 21, 567-572 (1994). 


\subsubsection{Principle of MOSFETs}

\section{Radiation response}

When a MOSFET is exposed to ionizing radiation, electron-hole pairs are created in the insulating silicon-dioxide layer. Some of the electrons will travel to the gate and some will recombine with the holes. The holes that have not recombined with the electrons will drift to the oxide-substrate interface where some of them will become trapped. The extra interfacial charge will cause a shift in the negative voltage that must be applied between the gate and source terminals to create the conducting channel and to achieve the same current flow as before the irradiation, as seen in Fig.2.2. This change in the threshold voltage, from before irradiation to after, $\Delta V_{t h}$, is proportional to the amount of dose delivered to the

\section{MOSFET.}

The sensitivity of a detector can be improved by increasing the amount of holes at the interface. This can be done by applying a positive gate bias during irradiation, which will increase the number of electrons collected at the gate, decreasing the amount of recombination and therefore increasing the number of positive holes leftover at the oxide-substrate interface. In addition, the positive gate bias pushes the holes toward the oxide interface. Another method is to increase the thickness of the oxide layer, which increases the number of electron-hole pairs created during irradiation; however, this improved sensitivity decreases the lifetime of the sensor. 


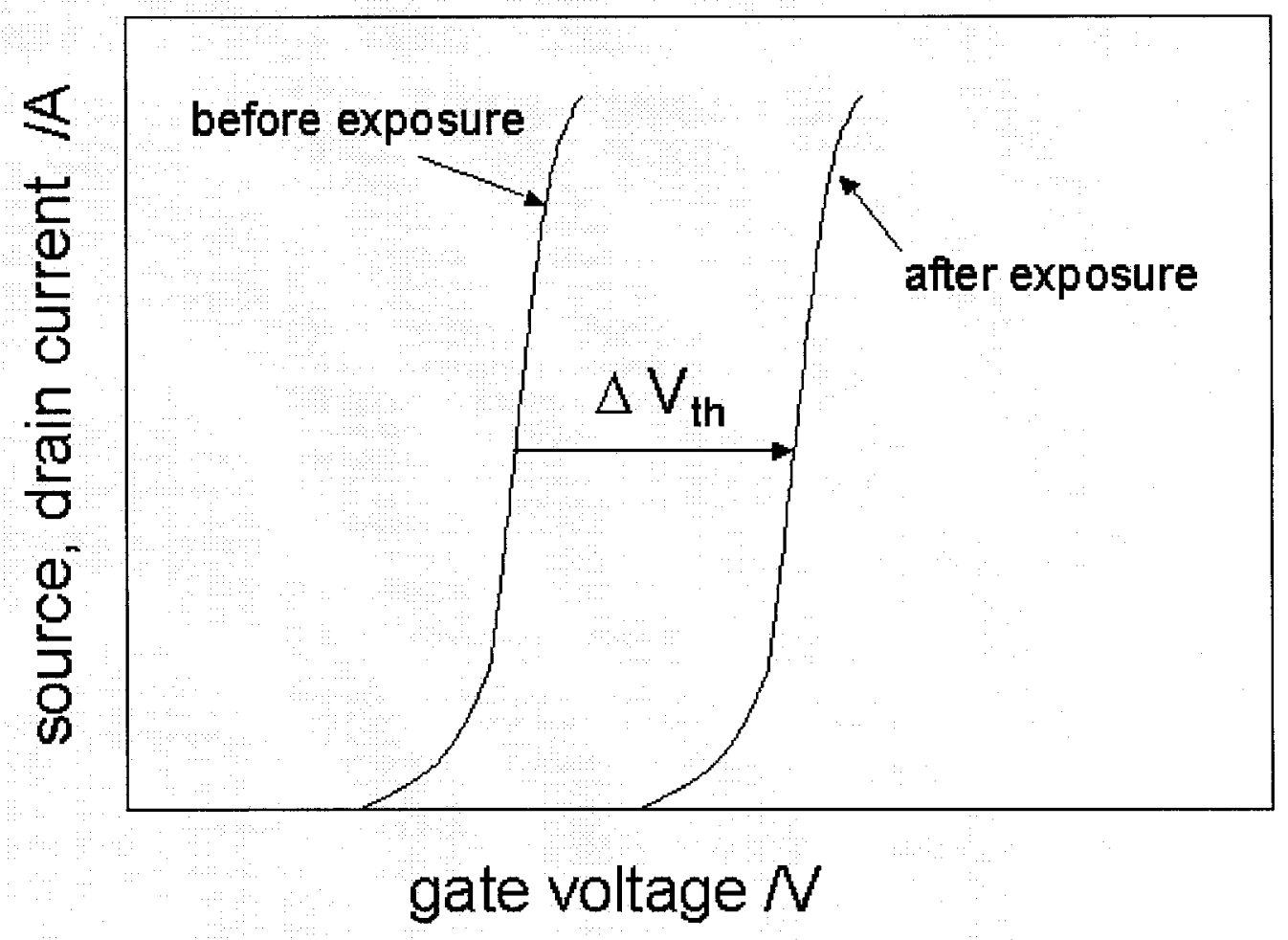

Fig.2.2 Change in threshold voltage with exposure to radiation. 


\subsubsection{Operation of MOSFET dosimetry systems}

For use as a dosimeter, a MOSFET is bonded to a long flexible cable with approximately $1 \mathrm{~mm}$ of epoxy, which acts as a guard against contamination, moisture, and heating. The epoxy creates a rounded bubble shape on one side of the detector, closest to the location of the oxide-substrate interface.

When the detector is positioned with this side facing the beam, it is said to be in 'bubble-up orientation', however the manufacturer recommended orientation of the MOSFET detectors is 'bubble-down orientation', where the epoxy-side is facing away from the beam, as shown in Fig.2.3.

a.
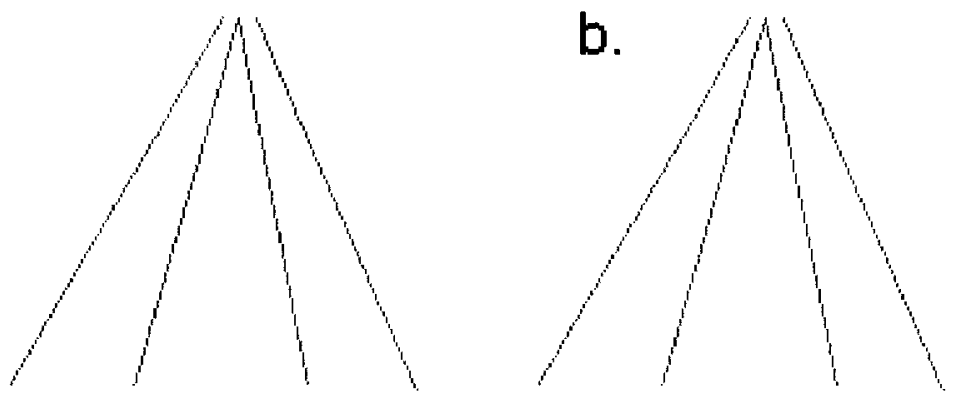

Fig.2.3.a. Bubble-up and b. bubble-down MOSFET orientations with respect to the beam. Diagram is not to scale. 
The cable is attached to a bias supply, which provides both the positive gate voltage during irradiation and the negative voltage necessary to determine the threshold voltage during the read-out. Five MOSFETs can be connected to the bias supply used in this work, each able to measure the dose at a desired position in the beam. The bias supply is connected to a reader that prints out the threshold voltage readings. In this work, a Standard Bias Supply (Model TN-RD20, Thomson Nielson, Division of Best Medical Canada LTD, Ottawa, ON) was used.

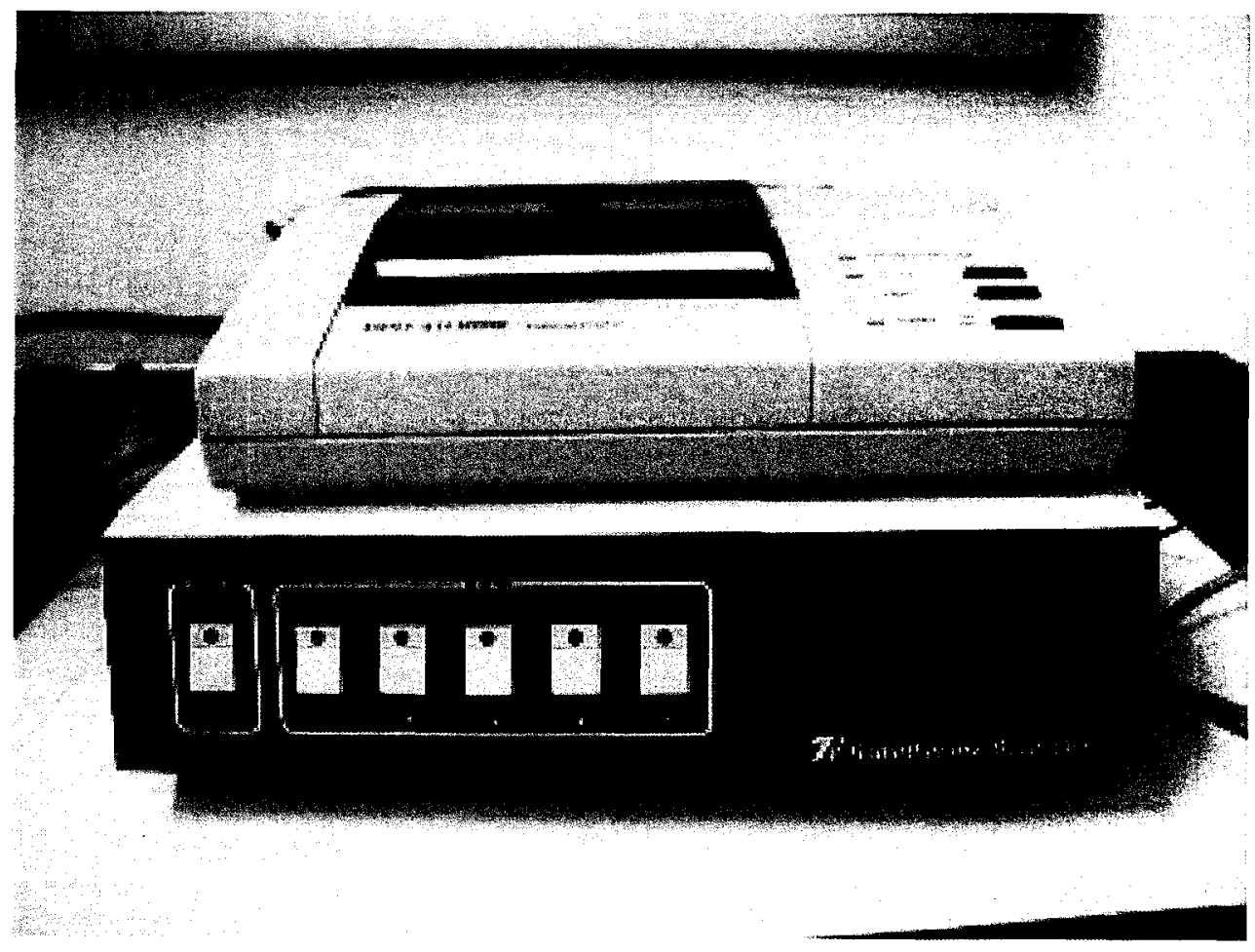

Fig.2.4 TN-RD-20 Standard Bias Supply and DPU-411 Type II Thermal Printer. 
To prepare for irradiation, the MOSFETs should be connected to the bias supply for approximately one hour before use to stabilize the system. The initial threshold voltage value for each MOSFET is read using the zeroing function of the reader. The bias supply can then be disconnected from the reader and transported along with the MOSFETs to the location of irradiation. Once the irradiation is complete, there should be a two-minute wait period before reading, which is suggested to ensure consistency of the readings. When the MOSFETs are read, the system will print out the total threshold voltage of each MOSFET in use. A previously determined calibration factor is then applied to the shift in threshold voltage $\left(\Delta V_{t h}=V_{t h}\right.$ after irradiation - $V_{t h}$ before irradiation) to calculate the absorbed dose of each detector.

\subsubsection{Types of MOSFETs available}

\section{Single bias Single MOSFET}

The first type of MOSFET proposed for in vivo dosimetry was a single bias, single MOSFET. The linear response of this detector breaks down with highaccumulated dose. This is due to a build up of positive holes in the oxide layer, which reduces the magnitude of the electric field created by the bias. This causes the electrons and holes to move more slowly across the conduction channel, allowing more time for recombination and thus decreasing the shift in $V_{t h}$ measured. This MOSFET also exhibits a significant dependence on temperature, due to the change in mobility of holes with temperature. A change in ambient temperature of 1 degree Celsius can cause a change in $V_{\text {th }}$ of as much as 4-5 $\mathrm{mV}$, which would be a $3 \%-6 \%$ effect for a typical skin dose measurement [10]. 
The effects of temperature are important to understand for in vivo dosimeters because the temperature of the detector during calibration is usually room temperature $\left(22^{\circ} \mathrm{C}\right)$, while the temperature can reach $37^{\circ} \mathrm{C}$ when in contact with a patient's skin.

\section{Dual bias Dual MOSFET}

The temperature dependence of single bias MOSFETs was improved by the design of the dual bias, dual MOSFET, which consists of two identical MOSFETs on a single chip. The two MOSFETs operate at two different biases and are irradiated simultaneously. The difference between the shifts in $V_{t h}$ of the two detectors $\left(\Delta V_{t h} 1-\Delta V_{t h} 2\right)$ is proportional to the total absorbed dose. Since both detectors are exposed to the same temperature conditions during calibration and in vivo measurements, using the difference of $\Delta V_{t h}$ and $\Delta V_{t h} 2$ reduces the effect of temperature on the overall response. It has been shown that in the range of 0 $80^{\circ} \mathrm{C}$, the dual bias, dual standard MOSFET has a temperature coefficient of only $0.015 \mathrm{mV} /{ }^{\circ} \mathrm{C}$. This detector also has better sensitivity, reproducibility and stability than the single MOSFET [10].

The first model of the dual bias dual MOSFET was a standard MOSFET (Models TN 502RD and TN 1002RD, Thomson Nielson, Division of Best Medical Canada LTD, Ottawa, ON), that has a physical size $3 \mathrm{~mm}$ long, $3 \mathrm{~mm}$ wide and $0.5 \mathrm{~mm}$ thick. A smaller model, microMOSFET, (Models TN 502RDM and TN 1002RDM) was later proposed by the same manufacturer to increase spatial resolution and decrease directional dependence. MicroMOSFETs have a physical size of 3.5 $\mathrm{mm}$ long, $1 \mathrm{~mm}$ wide, and $0.5 \mathrm{~mm}$ thick and a sensitive volume $0.2 \mathrm{~mm}$ long, 0.2 
$\mathrm{mm}$ wide and 0.5-1 $\mu \mathrm{m}$ thick. Because of the smaller size of the microMOSFETs, they are more suitable for use in high dose gradients. All MOSFETs used in the work discussed in this thesis were microMOSFETs.

MOSFETs also come in two distinct sensitivity levels, high sensitivity (models TN 1002RD and TN 1002RDM) and standard sensitivity (models TN 502RD and TN 502RDM). The calibration factors quoted by the manufacturer are $0.33 \mathrm{cGy} / \mathrm{mV}$ and $1.00 \mathrm{cGy} / \mathrm{mV}$ for high and standard sensitivity MOSFETs, respectively, measured in a Co-60 beam. The high sensitivity MOSFETs have a thicker oxide layer as mentioned above. This makes high sensitivity MOSFETs ideal for in vivo measurements, where measured doses are often less than 2 Gy, but decreases the lifetime of the MOSFET since it will reach its saturation level after a smaller accumulated dose. Both high sensitivity and standard sensitivity MOSFETs are used throughout this work, but from now on the term MOSFET will be used to represent a dual bias, dual microMOSFET.

Some researchers have altered the thickness of the epoxy used in MOSFET dosimeters to make a thinner detector, with minimal build-up. This was done to measure doses closer to the actual patient surface. The reproducibility of the sensor response was within $2 \%$, however there was significant variation within a batch of MOSFETs. This is due to the manufacturing of the detectors not being perfect, since any variation in the thickness of the epoxy has a strong effect due to the steep dose gradient at small depths [11]. 


\section{Implantable MOSFET}

There are also implantable MOSFETs, which are designed for permanent implantation within the body. These MOSFETs are sealed airtight in packages to prevent contamination or damage when placed within the human body. The detectors are read telemetrically and can be used to take multiple readings throughout the course of external beam radiotherapy treatment as well as act as fiducial markers. These MOSFETs are rigorously calibrated and have shown agreement with ion chamber measurements within $\pm 3.5 \%$ [12]. Trials have shown no adverse effects due to the placement of the detector in the tumour and researchers have been successful in taking daily readings of tumour doses for various types of cancer [13].

\subsubsection{Characteristics of microMOSFETs}

\section{Dosimetric property}

The dosimetric properties of microMOSFETs in conventional radiation beams have been studied extensively, and results show that they perform within the acceptable limits of clinical in vivo dosimeters. It has been reported that microMOSFET response to dose is linear for doses up to 5 Gy $[7,14]$. The recommended lifetime of a MOSFET is $18,000 \mathrm{mV}[14,15]$. At this point, the sensitivity of the detector can decrease by $5 \%$ [15] as the number of holes in the oxide layer reaches saturation.

The MOSFET reader used in these experiments displays a warning message when the detector is reaching saturation, advising the user to change detectors. Once the MOSFET reaches an unusable state, the system prints an error 
message rather than a $V_{t h}$ value. It has also been shown that microMOSFETs are dose rate independent, in the dose rate range from 100 to $600 \mathrm{MU} / \mathrm{min}$, Fig.2.5 [14].

\section{Creep-up effect}

As mentioned above, there is a dependence on the time the MOSFET is read relative to the irradiation. When a MOSFET is read, the measuring circuit injects a current into the detector, which temporarily disturbs the charge distribution.

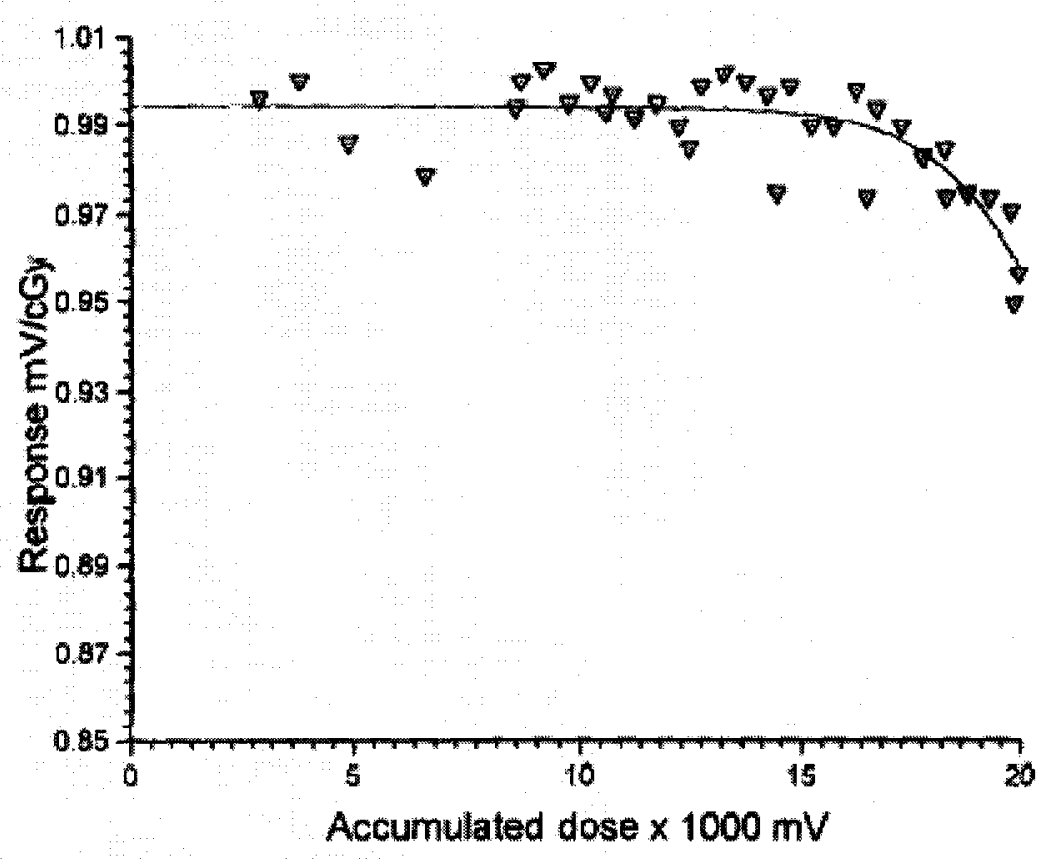

Fig.2.5 Effect of accumulated dose on the relative response of a standard MOSFET sensor. Graph from R Ramaseshan, S Russell, and P O'Brien, "Clinical dosimetry using MOSFETs" Int. J. Radiat. Onc. Biol. Phys. 1997;37,959-964 [15]. 
This effect decays during the first minute after a reading, however it can be amplified if another reading is taken before if has fully decayed. This is known as the creep-up effect, and can cause the $V_{\text {th }}$ of the MOSFET to increase with consecutive readings depending on the time between the readings, Fig.2.6. This is a significant effect which can cause a maximum increase in $V_{t h}$ of $4 \mathrm{mV}$, which corresponds to a dose of 4 cGy for a MOSFET with a sensitivity of $1.00 \mathrm{mV} / \mathrm{cGy}$ [15].

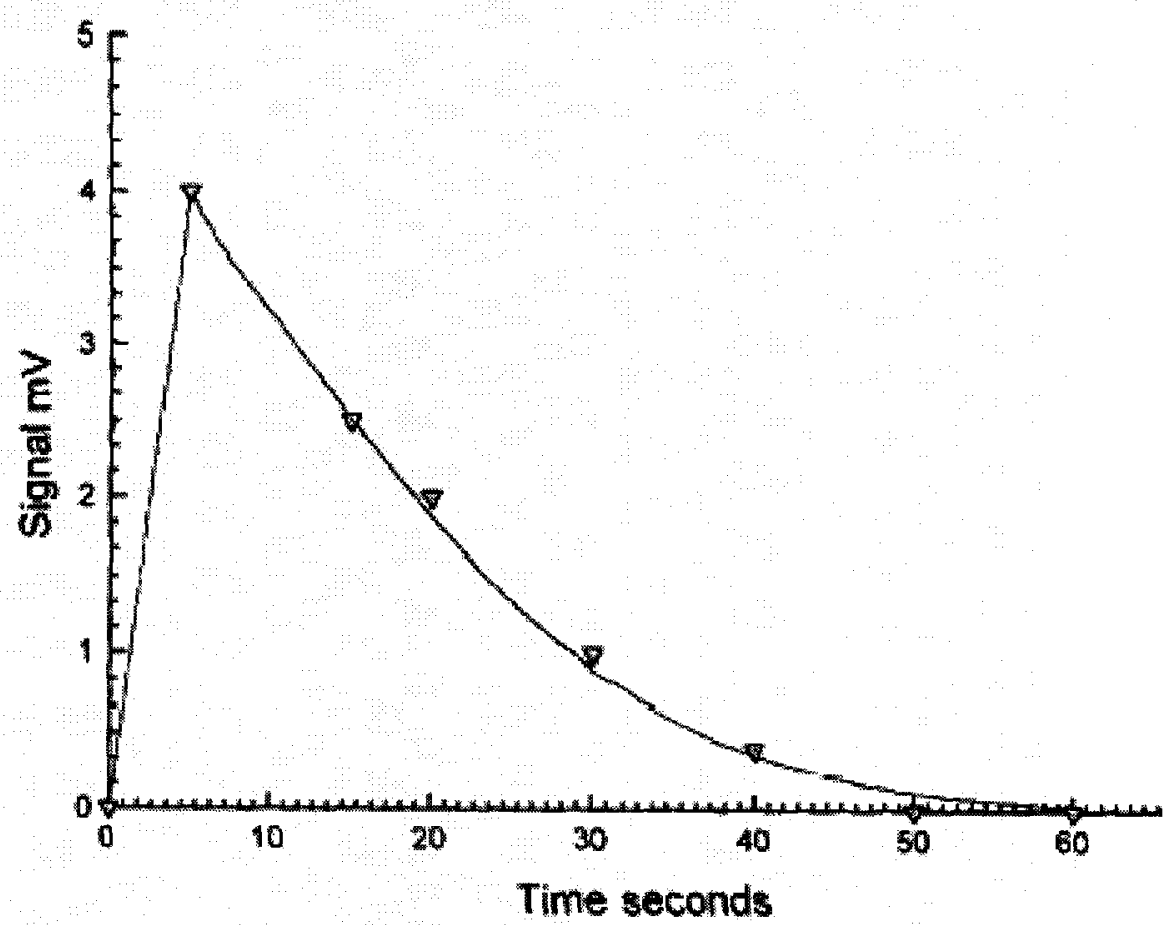

Fig.2.6 The MOSFET signal with consecutive readings plotted versus time after initial reading, a phenomenon known as "the creep-up effect". Graph from R Ramaseshan, S Russell, and P O'Brien, "Clinical dosimetry using MOSFETs" Int. J. Radiat. Onc. Biol. Phys. 1997; 37: 959-964 [15] 
MOSFET $V_{\text {th }}$ signals can also fade by up to $3 \%$ within the first 5 hours after exposure, and after this period, the signal remains stable for up to 60 hours postirradiation [14].

\section{Directional and energy dependence}

The directional dependence of the standard MOSFETs is reported as $\pm 2.5 \%$ $[7,15]$ when measured with full build-up in a $6 \mathrm{MV}$ beam. The main overresponse occurred between gantry angles of $140^{\circ}$ and $220^{\circ}$, where $0^{\circ}$ corresponds to a bubble-down orientation. This dependence is increased when measured on the surface, where there is a lack of charged particle equilibrium. Under these conditions, a variation of up to $7 \%$ was noted between angles $25^{\circ}$ and $80^{\circ}[7,15]$. An improved dependence of only $\pm 2 \%$ was measured with the microMOSFET under full build-up, likely due to the reduction in the thickness of the silicon substrate layer [14]. In the therapy energy range between $4 \mathrm{MV}$ and $25 \mathrm{MV}$, the microMOSFET response was uniform within $\pm 2-3 \%$, a significant improvement from the standard MOSFET [14].

\section{Reproducibility and Accuracy}

Another performance characteristic of detectors is reproducibility, which is the comparison of the readings under fixed conditions. MOSFETs have been shown to have a reproducibility of $3 \%$ for doses characteristic of patient treatments $[15,16]$. As the standard deviation depends on the magnitude of the $V_{t h}$ shift, the reproducibility can be improved by using high sensitivity MOSFETs, which have a larger $\Delta V_{t h}$ for the same dose. MOSFET measurements agree with ion chamber measurements made in a solid water phantom under a static beam within $2 \%$ 
[15]. When used on patients for in vivo dosimetry, MOSFET measurements can be compared with treatment plans, or other dosimeters to evaluate the accuracy of the measurements. Surface dose measurements on patients showed a standard deviation of $3.9 \%$ between standard MOSFET readings and the calculated expected dose [15]. MicroMOSFET measurements made on the surface of an anthropomorphic phantom showed agreement within $2 \%$ between readings and a parallel-plate chamber [14]. One of the biggest advantages of MOSFETs over other in vivo dosimeters is the small size, which lends itself well to use in IMRT or brachytherapy, where high dose gradients are present. Measurements made on patients with oropharynx and nasopharynx cancers being treated with IMRT showed agreement between MOSFETs and calculated dose of $5 \%$. This included errors due to inter-fraction patient positions, intrafraction patient movement and also due to variation in MOSFET positioning [8]. Other work [7] has shown deviations of $5 \%$ between MOSFET surface dose measurements made on an anthropomorphic phantom and an IMRT treatment planning system. This same study also showed a variation of $3 \%$ between the treatment plan doses and ion chamber measurements. Treatment planning systems are known to have problems calculating doses on and near the surface, as will be discussed later on, so the inaccuracy of the MOSFET measurements is most likely over-estimated. 


\subsection{Thermoluminescence dosimeters (TLDs)}

\subsubsection{Physics of TLDs}

One of the most commonly used thermoluminescent materials is lithium fluoride, $\mathrm{LiF}$, because it has an effective atomic number of 8.31 , which is very close to that of tissue, 7.22. The most common impurities added to the TLD material are $\mathrm{Mg}, \mathrm{Ti}$ and $\mathrm{Mg}, \mathrm{Cu}, \mathrm{P}$. When such impurities are added to the material, energy traps are created in the forbidden region between the LiF valence and conduction bands. When the material is irradiated, energy is absorbed from the secondary charged particles that lifts electrons out of the valence band and into the conduction band. Most of these electrons return directly to the valence band, releasing their excess energy by emitting photons through a process called fluorescence. Some of the electrons can alternatively be trapped in one of the metastable impurity energy levels.

When the irradiated material is heated, the trapped electrons receive enough energy to lift them out of the traps and into the conduction band. The electrons then return directly to the valence energy band with the emission of light through thermoluminescence. The amount of light emitted is proportional to the number of trapped electrons, which is proportional to the amount of the absorbed radiation dose. The thermoluminescence intensity emission is measured with a photomultiplier tube, PMT, and, when graphed as a function of TLD temperature, creates a 'glow curve', Fig.2.7. The different peaks on the curve represent different trapped energy levels. By heating the TLDs at a lower temperature (100 ${ }^{\circ} \mathrm{C}$ ) for ten minutes before reading, the electrons trapped in the lower unstable 
energy traps receive enough energy to be lifted out of the traps and into the conduction band, and then returned to the valence energy band, leaving only the electrons of interest to produce light during reading. The absorbed dose can then be measured by determining the area under this curve.

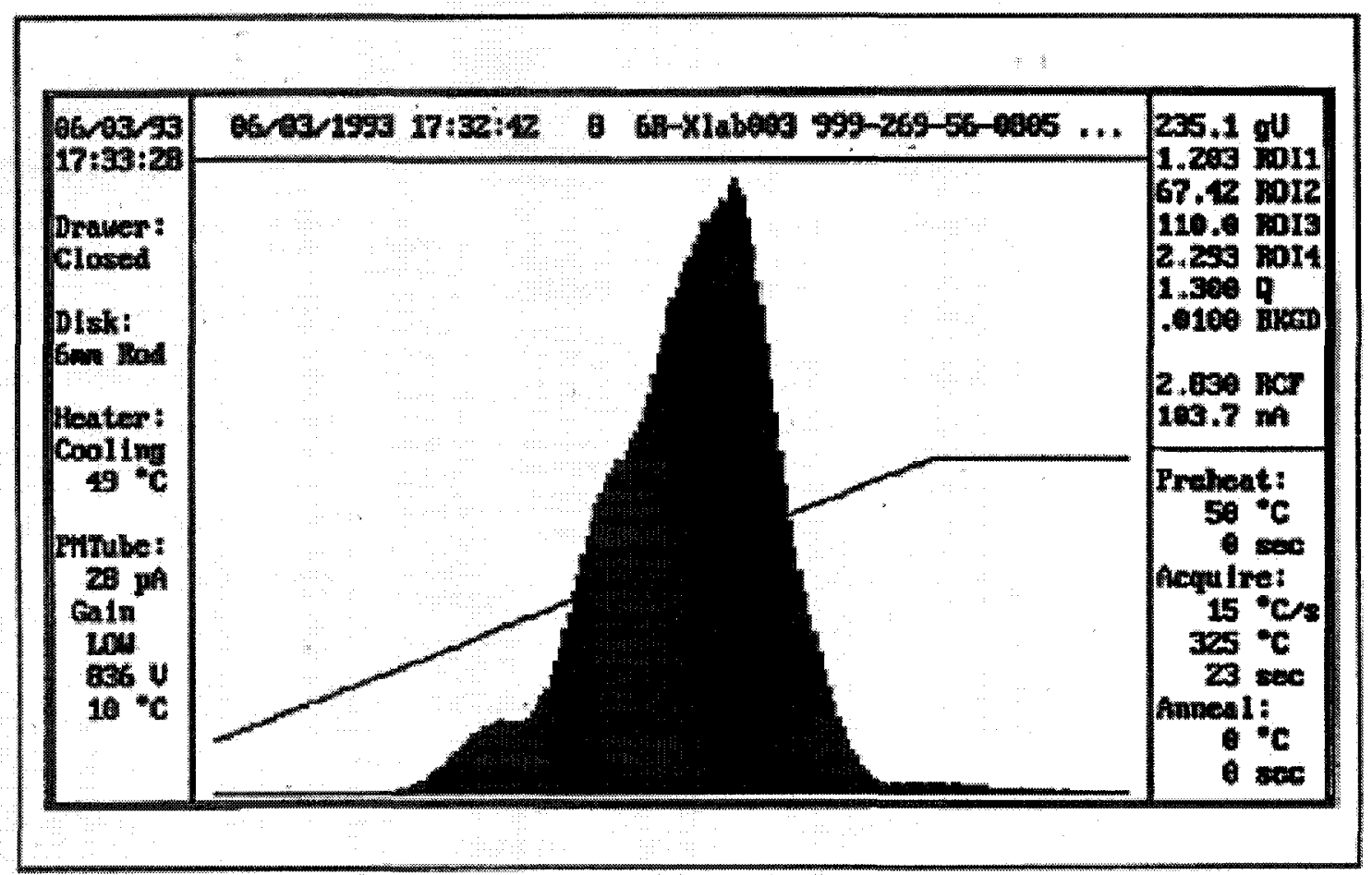

Fig.2.7 A typical thermogram (glow curve) of a LiF:Mg,Ti TLD chip as measured by a Harshaw TLD reader. 
Before each use, the TLDs must be annealed by heating them at a temperature of $400^{\circ} \mathrm{C}$ for one hour and then at $100^{\circ} \mathrm{C}$ for two hours, allowing them to cool in between. This process erases any residual signal from previous irradiations. For use as in vivo dosimeters on patients during treatment, TLD chips are enclosed in small bandages. The chips are encased in vacuum-sealed pockets of plastic wrap and attached to strips of tape. Each bandage is numbered to keep track of the chips. After irradiation, TLD chips are carefully removed from the bandages and read.

\subsubsection{Advantages and Disadvantages of TLDs}

TLDs don't require biasing or other wires, making measurements and transport convenient. They can also store dose information for long periods of time before reading, which makes them suitable for mailing and allows for comparison between different institutions. TLDs are tissue-equivalent and studies have shown that they have a small dependence on dose rate, temperature and energy in the therapeutic range [17]. Carefully calibrated TLDs can have a reproducibility of $2 \%$ [18], however for the best results, they must be calibrated frequently and processed with great care. The result is that the general use of TLDs is quite time consuming. Other studies have shown a reproducibility of $\pm 5 \%$ when the processing time is more practical for general routine dosimetry [18]. Because TLD chips have a significant thickness $(1 \mathrm{~mm})$ a useful technique to determine surface dose is to make several measurements using TLDs of various thickness, and extrapolate to the surface [19]. TLD chips are approximately $2.5 \times 2.5 \times 1.0$ $\mathrm{mm}^{3}$ in size and therefore are not ideal for point dose measurements. In a study 
comparing in vivo measurements with expected dose, the TLD measurements had a difference of $5.1 \%$, while the MOSFET measurements had a difference of $3.9 \%[15]$.

\subsection{Diodes}

\subsubsection{Physics of Diodes}

Diode dosimeters consist of a p-n junction silicon diode. The silicon base material can be either n-type or p-type, however the p-type material is less affected by radiation damage and is therefore the only type used for dosimetry. The surface of the silicon base is doped with material of the opposite type, which in this case would be n-type. When a diode is exposed to radiation, electron-hole pairs are created and the minority charge carriers are swept into the depletion region. The presence of an electric field causes these charges to move across the depletion region, creating a current. The magnitude of this current is linear with radiation dose and can be measured during irradiation to provide online dose information.

\subsubsection{Characteristics of Diodes}

Diodes have been used for in vivo dosimetry since the 1980's. They have been shown to give results identical to TLDs when measuring entrance and exit doses, but TLDs can have better reproducibility when careful processing and calibration procedures are followed [20]. Diodes are still often preferred over TLDs since they provide online readouts, however this necessitates a connection to the reader during the entire treatment, which makes set-up and cables cumbersome. A wireless diode system has recently been introduced by Sun Nuclear that 
transmits dose information to a wall transponder via radio frequency (Sun Nuclear Corporation, Melbourne, FL). The reproducibility of this system is $0.5 \%$ and the angular dependence is $1.0 \%$, as measured by the manufacturer. Diodes cannot store dose information and their large size $\left(0.8 \times 0.8 \mathrm{~mm}^{2}\right)$ makes them unsuitable for measurements in regions of high dose gradients, such as in IMRT. Some studies [16] have shown that diodes have an intrinsic precision of $0.05 \%$ compared to $0.7 \%$ for MOSFETs for in vivo patient measurements, however the total uncertainty in entrance dose determination was $2.9 \%$ for diodes and only $2.0 \%$ for MOSFETs. Diodes also showed a significantly larger dependence on temperature, dose rate, accumulated dose, and energy than MOSFETs.

\subsection{Optically Stimulated Luminescence Dosimeters (OSLs)}

Optically stimulated luminescence dosimeters, OSLs, consist of carbon-doped aluminum oxide. They are very similar to TLDs except that the trapped electrons are stimulated to produce luminescence by the application of light instead of heat. After irradiation, a laser is used to excite the OSL chip and the resulting light is measured by a PMT. OSLs have an advantage over TLDs in that they can be read several times and have an increased sensitivity. Studies have shown that OSLs have a reproducibility of $0.7 \%$ [21] and a dependence on dose rate, field size and energy within $1 \%$. Angular dependence of OSLs is affected by the shape of the detector, but has been shown to be less than $2 \%$ [22]. They have been widely used for personal dosimetry; however they are not currently used or 
manufactured specifically for in vivo dosimetry during radiation therapy treatments. 


\section{Chapter 3}

\section{IMRT and Tomotherapy}

The goal of any treatment plan is to deliver adequate dose to target volumes while sparing healthy tissue. The consequences of delivering dose to certain healthy organs are more severe than for others. Depending on the location of the tumor, physicians will identify and contour specific organs at risk, OARs, which should have special consideration in terms of dose limits. The use of conventional uniform beams places strict constraints on treatment planning, as it is limiting on the sparing of OARs that are in close proximity to the target volume. Multiple beams, which enter the body from various angles, can be used to allow for some sparing of critical organs, however the target dose is sometimes still constrained to levels lower than ideal in order to limit adverse effects in healthy tissues. Many advancements have been made in the delivery of radiation therapy in recent years to address these problems, including the introduction of intensity modulated radiation therapy in the early 1990's [2] followed by the development of helical tomotherapy [3].

\subsection{Intensity Modulated Radiation Therapy}

Intensity modulated radiation therapy allows for increased sparing of organs at risk through the optimization of beams with nonuniform radiation fluences. Multileaf collimators are used to deliver conformal dose profiles that have been optimized through a process called inverse treatment planning. In conventional radiotherapy, the treatment plan is constructed using forward planning, whereby the planner uses different beam angles, weights, and wedges to try to achieve a 
dose that matches the prescription as closely as possible. Alternatively, in IMRT, inverse planning is used, whereby a dose is prescribed and constraints applied, and then an optimization program is used to find the beam parameters that can best achieve these goals [23].

During optimization, there is a trade-off between delivering the optimal dose to the targeted structures and minimizing the dose to the organs at risk. Once all structures are outlined and the clinical constraints are introduced, weighting factors are assigned to the constraints to determine the level of importance of satisfying each specific constraint. A small weighting factor would mean only a small penalty if the constraint wasn't satisfied, while a larger weighting factor would give the constraint higher priority for satisfaction. A computer then carries out the optimization procedure and produces curves showing the dose delivered to various organs at risk, OARs, and tumor volumes. IMRT gives much more flexibility to treatment planning, allowing for significantly higher dose to targets, while at the same time sparing critical organs [23].

IMRT became increasingly popular with the development of commercial planning and delivery software from all major Linac manufacturers, starting with the PEACOCK system introduced by the NOMOS Corporation (Swickley, Pennsylvania, USA) [24]. One of the main downfalls of IMRT is the increased dependence on consistent geometry of the tumor and surrounding organs because of the high dose gradients in the treatment plans. Because of this, many of the systems currently being installed also incorporate image guidance, which allows the user to take an image of the patient while on the treatment couch at 
the time of delivery. This process can identify changes in internal geometry and can remove uncertainties in tumor position thus reducing delivery errors [25].

\subsection{Tomotherapy}

The latest technology in radiation therapy is helical tomotherapy, which delivers intensity modulated radiation in a helical pattern as the treatment couch moves through the bore of the machine [3]. The idea of Tomotherapy was first explored in the early nineties by a research group at the University of Wisconsin and the first patient was treated on a commercial Tomotherapy Hi-art unit (TomoTherapy Inc., Madison, Wisconsin, USA) in 2003 [26].

The Tomotherapy unit consists of a linear accelerator mounted on a gantry, which is used to deliver the prescribed dose as well as for image acquisition. As the patient moves through the bore, the linac rotates, delivering dose in a helical pattern. The beam is split into thousands of tiny beamlets, which are intensity modulated using binary leaves that can be either open or closed [26]. Inverse treatment planning is used; however, with Tomotherapy, the degrees of freedom are greatly increased, giving more control over treatment delivery. The number of beam angles is greatly increased from IMRT with a linac, Fig.3.1, allowing for an increased conformity of the dose to the target, and the treatment is delivered continuously, which has radiobiological advantages. 
a.

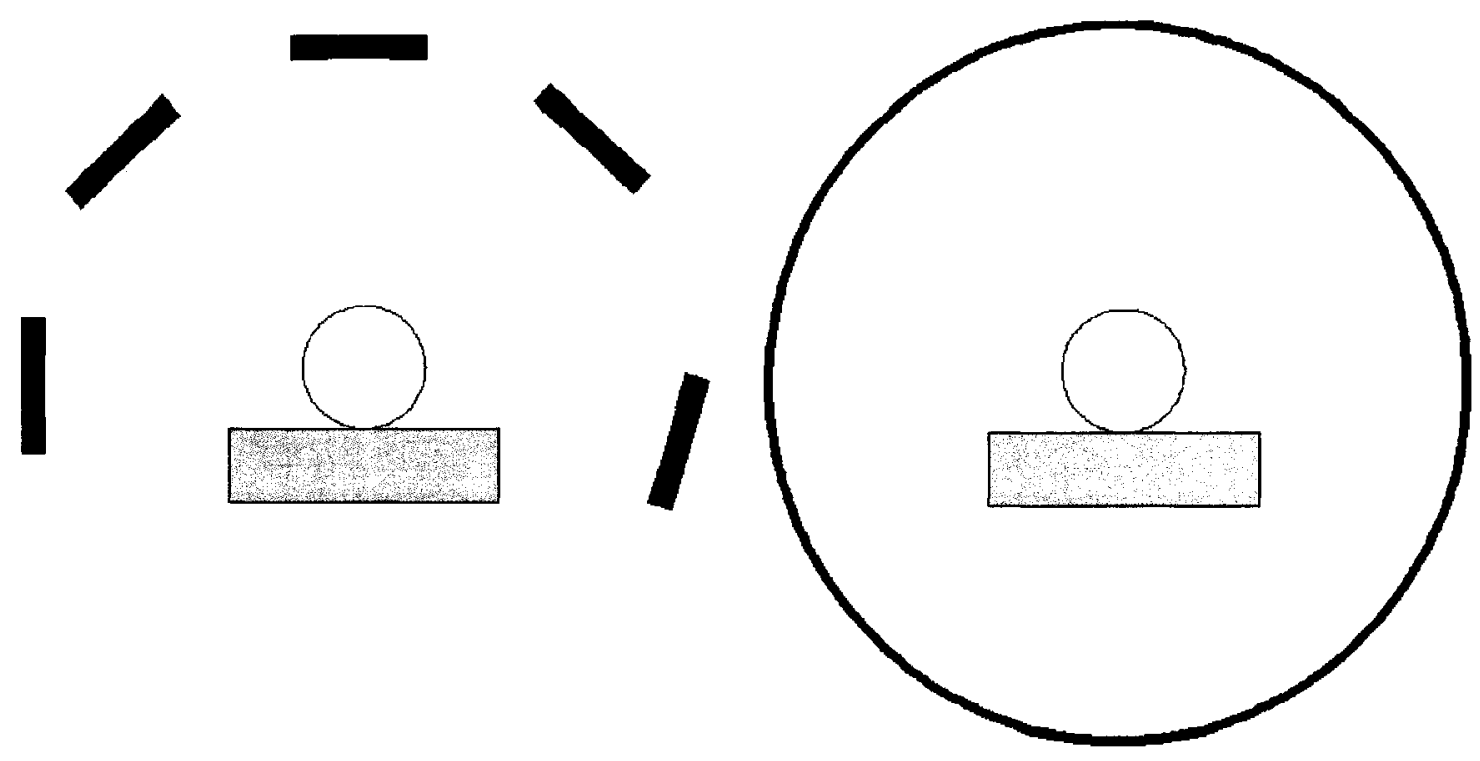

Fig.3.1 Diagram showing the geometry for a. IMRT and b. helical Tomotherapy treatment delivery. In each case, the beam (shown in blue) is intensity modulated, however, for IMRT, treatment is delivered from only a few angles, while with tomotherapy, the dose is delivered from a full $360^{\circ}$ as the patient (represented by yellow circle) moves through the bore of the machine.

Since the dose delivered with helical tomotherapy is highly conformal, accurate positioning is particularly important. At the start of each treatment, the patient is positioned on the couch and imaged using Mega-voltage computed tomography, MVCT. The daily image is compared to the original kVCT image set that was used for treatment planning and adjustments are made as necessary to reproduce the tumor position as accurately as possible before the treatment is delivered. 


\section{Chapter 4}

\section{Characterization of MOSFETs used in this study}

\subsection{MOSFET Calibrations}

\subsubsection{Calibrations on a 6 MV LINAC}

In this research, dual-bias-dual standard sensitivity and high sensitivity microMOSFETs (Models TN-502RDM and TN-1002RDM respectively, Thomson Nielsen, Division of Best Medical Canda, Ottawa, ON) were used. Before the MOSFETs were used for dose measurements they were first calibrated in a $6 \mathrm{MV}$ linear accelerator beam. Calibration factors were determined for both high and standard sensitivity MOSFETs. The MOSFETs were connected to a $5 \mathrm{~V}$ standard bias supply at least one hour before calibrations were done. The bias supply was also connected to a reader, which provided a printout of the threshold voltage of each MOSFET. One at a time, each detector was positioned at a depth, $d_{\text {MOSFET }}$, of $5 \mathrm{~cm}$, in a solid water phantom and a $10.3 \mathrm{~cm}$ slab of solid water was used for backscatter. The calibrations were done in an source-to-surface distance, SSD, setup at an SSD of $100 \mathrm{~cm}$. For bubble-up calibrations, the MOSFET was placed with the epoxy bubble facing towards the beam and for bubble-down calibrations the epoxy bubble was facing away from the beam. A Farmer type ionization chamber, type NE 2571, Nuclear Enterprises, U.K., which had a volume of 0.6 $\mathrm{cc}$, was positioned with its center at a depth, $d_{\text {ionchamber }}$, of $11.3 \mathrm{~cm}$ in the solid water phantom, $6.3 \mathrm{~cm}$ below the MOSFET location, Fig.4.1. 


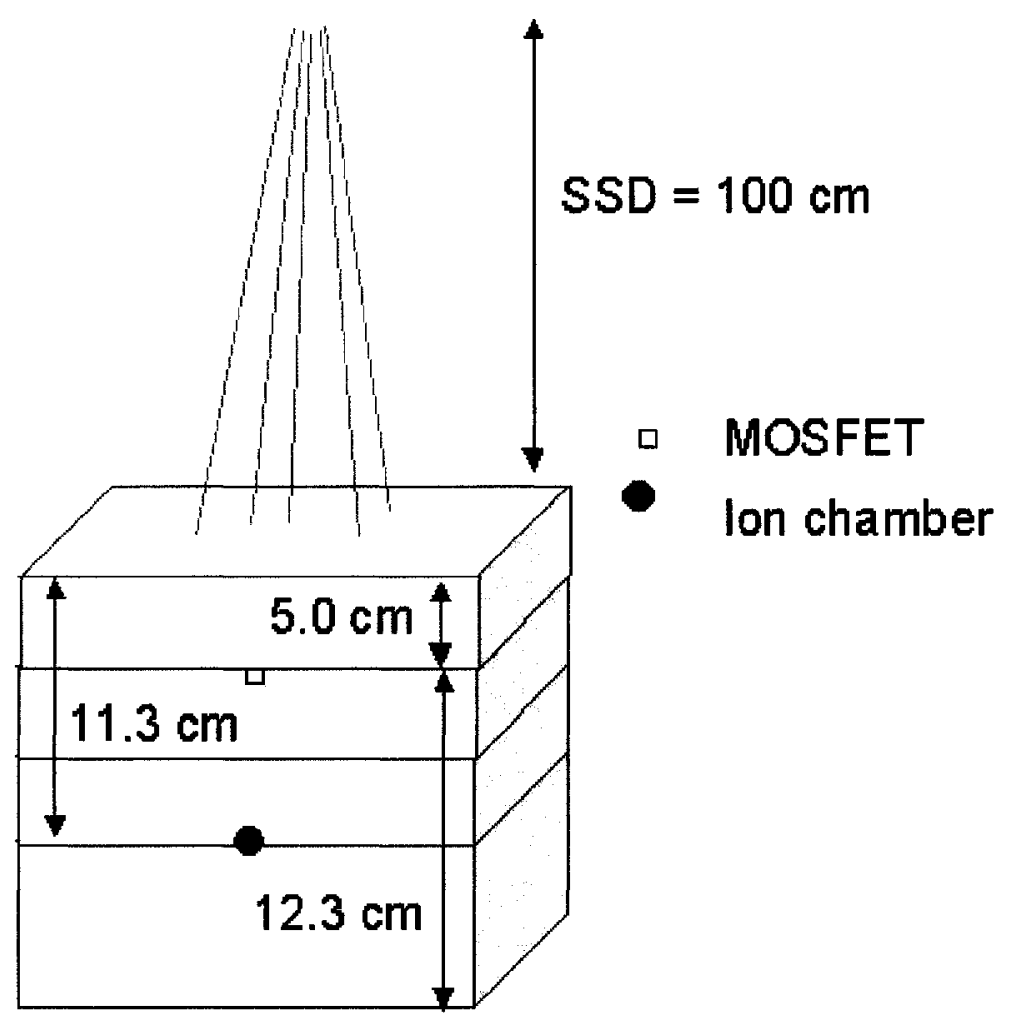

Fig.4.1 Experimental set-up for MOSFET calibrations in a conventional linac beam. 
After an initial reading of the threshold voltage was taken, 50 monitor units were delivered at a field size of $10 \times 10 \mathrm{~cm}^{2}$. The threshold voltage was read again two minutes after the irradiation. This process was repeated three times for each calibration. Ion chamber readings were taken during each trial, and the absorbed dose to water at the depth of the ion chamber, $D\left(d_{\text {ionchamber }}\right)$, was calculated using the following equation from AAPM's TG-51 protocol [47]:

$$
D_{w}^{Q}=M k_{Q} N_{D, w}^{60} \mathrm{Co}
$$

In this equation, $M$ is the fully corrected ion chamber reading at the reference depth, $N_{D, w}^{60} C_{0}$ is the absorbed dose to water calibration coefficient provided by the NRC and $k_{Q}$ is the quality conversion factor. Several factors were applied to the raw ion chamber readings, $M_{\text {raw }}$, to account for effects of the polarity of the electrometer voltage, ion recombination, and the pressure and temperature of the air within the chamber. The correction factors for polarity, $P_{p o l}$, and ion recombination, $P_{i o n}$, were previously determined for the specific ion chamber and beam used. The pressure and temperature was measured during each set of calibrations and the correction factor, $P_{T P}$, was calculated each time using:

$$
P_{T P}=\frac{273.2+t}{273.2+22.0} \times \frac{101.33}{P}
$$

This correction factor corrects for changes in the mass of air inside the chamber with variations in temperature and pressure. Percent-depth-dose curves that had been measured using a photon diode, $P D D_{\text {diode }}(d)$, were used to calculate the dose delivered at the depth of the MOSFET detector. 


$$
D\left(d_{\text {MOSFET }}\right)=D\left(d_{\text {ion chamber }}\right) \times\left[\frac{P D D_{\text {diode }}\left(d_{\text {MOSFET }}\right)}{P D D_{\text {diode }}\left(d_{\text {ion chamber }}\right)}\right]
$$

Comparing the change in threshold voltage, $\Delta V_{t h}$, with the calculated dose delivered, $D\left(d_{\text {MOSFET }}\right)$, a calibration factor, $C F(\mathrm{cGy} / \mathrm{mV})$, was determined for each detector.

$$
C F(c G y / m V)=\frac{D\left(d_{\text {MOSFET }}\right) c G y}{\Delta V_{t h} m V}
$$

\subsubsection{Calibrations in Cobalt-60 beam}

To compare calibration factors at different beam energies, several MOSFETs were also calibrated in a Cobalt-60 beam. The experimental set-up was similar to that in the $6 \mathrm{MV}$ beam, with the MOSFETs at a depth of $5 \mathrm{~cm}$ and an ion chamber positioned at a depth of $11.3 \mathrm{~cm}$ in a solid water phantom. The calibrations were done in an SSD set-up, at an SSD of $80 \mathrm{~cm}$ and in a field size of $10 \times 10 \mathrm{~cm}^{2}$. Each MOSFET was irradiated three times with a dose of $50 \mathrm{cGy}$ at $d_{\max }$ and the ion chamber measurements and $P D D_{\text {diode }}(d)$ curves were used to determine calibration factors as described above. A total of 15 standard sensitivity MOSFETs and 14 high sensitivity MOSFETs were calibrated both face-up and face-down in the Cobalt-60 beam.

\subsubsection{Calibration factor dependence on field size and accumulated dose}

The total lifetime of a MOSFET detector is approximately $18,000 \mathrm{mV}$. To observe any change in sensitivity with total accumulated dose, one high sensitivity MOSFET and four standard sensitivity MOSFETs were calibrated at various 
points throughout their lifetime. All calibrations were done in a $6 \mathrm{MV}$ beam, at an SSD of $100 \mathrm{~cm}$ and in a field size of $10 \times 10 \mathrm{~cm}^{2}$ at a depth of $5 \mathrm{~cm}$.

The relationship between field size and calibration factor was also explored. For this purpose, one high sensitivity MOSFET and three standard sensitivity MOSFETs were calibrated at an SSD of $100 \mathrm{~cm}$ in a $6 \mathrm{MV}$ beam at various field sizes ranging from $10 \times 10 \mathrm{~cm}^{2}$ to $25 \times 25 \mathrm{~cm}^{2}$. The high sensitivity MOSFET had no radio-opaque markers while the three standard sensitivity MOSFETs had zero, one and two markers.

\subsubsection{Calibrations on Tomotherapy Unit}

The physical constraints of the Tomotherapy unit, such as the lack of a flattening filter and the limitations of the jaws, do not allow for the set-up of ideal calibration conditions. Therefore, MOSFETs used in this study for in vivo measurements on patients treated with Tomotherapy were calibrated with a conventional $6 \mathrm{MV}$ linac. To evaluate the accuracy of these calibration factors when used with the Tomotherapy unit, several MOSFETs were calibrated on both the Tomotherapy unit and the $6 \mathrm{MV}$ linac beam for comparison. One high sensitivity MOSFET and three standard sensitivity MOSFETs were calibrated with the Tomotherapy unit at an SSD of $80 \mathrm{~cm}$ and in a field size of $5 \times 40 \mathrm{~cm}^{2}$, Fig.4.2. Each MOSFET was placed at a depth of $1.5 \mathrm{~cm}$ in a solid water phantom, and $22 \mathrm{~cm}$ was used for backscatter. A Farmer ion chamber was placed at the same depth as to the MOSFET to measure the dose delivered during each trial. An average dose of $801.1 \pm 0.7$ cGy, as measured by the ion chamber, was delivered during each 
calibration by a stationary beam. A calibration factor was then calculated for each MOSFET as described above.

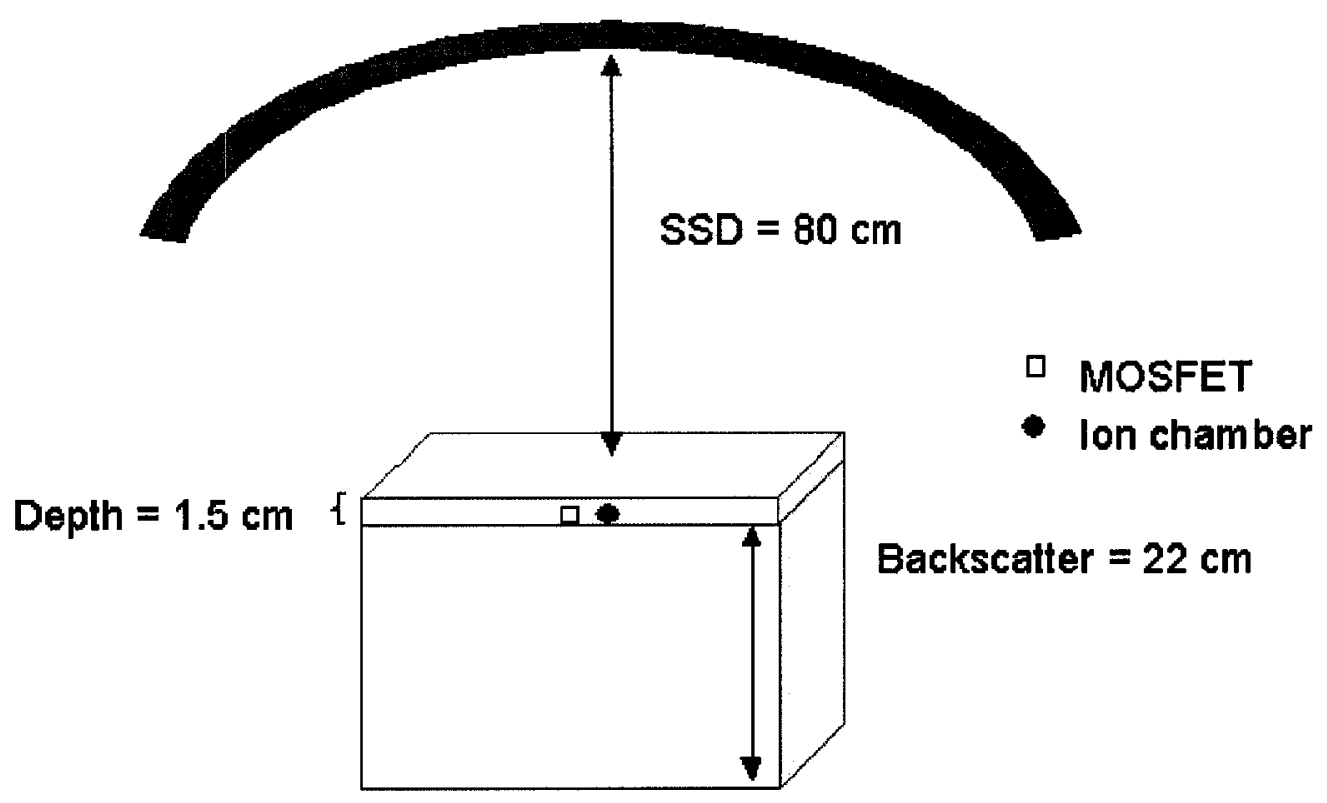

Fig.4.2 Experimental set-up for MOSFET calibrations using a Tomotherapy hi-art unit beam. 


\subsubsection{Results}

The results of the calibrations in a $10 \times 10 \mathrm{~cm}^{2}$ field size in both $6 \mathrm{MV}$ and Cobalt60 beams can be seen in Tables 4.1 and 4.2. The uncertainty shown is the standard deviation of the three measurements. The calibration factors quoted by the manufacturer are $0.33 \mathrm{cGy} / \mathrm{mV}$ and $1.00 \mathrm{cGy} / \mathrm{mV}$ for high and standard sensitivity MOSFETs, respectively, measured in a Co-60 beam. Our average calibration values for the high sensitivity MOSFETs of $(0.345 \pm 2.5 \%) \mathrm{cGy} / \mathrm{mV}$ in a $6 \mathrm{MV}$ beam, and $(0.345 \pm 0.69 \%) \mathrm{cGy} / \mathrm{mV}$ in a Co-60 beam were slightly above the manufacturer quoted value of $0.33 \mathrm{cGy} / \mathrm{mV}$. The average values for standard sensitivity MOSFETs of $(0.901 \pm 2.4 \%) \mathrm{cGy} / \mathrm{mV}$ in a $6 \mathrm{MV}$ beam and $(0.887 \pm$ $1.2 \%) \mathrm{cGy} / \mathrm{mV}$ in a Co-60 beam were slightly below the manufacturer quoted value of $1.00 \mathrm{cGy} / \mathrm{mV}$. The average calibration factors are identical for both energies within the uncertainty values quoted. Both groups of detectors had a slightly higher calibration factor when positioned in the bubble-down orientation, $t=-3.36, p=0.002$ for the standard sensitivity MOSFETs and $t=-5.71, p<0.001$ for the high sensitivity MOSFETs, however the difference between the two calibration factors for any individual detector was small. The reproducibility of the average energy, sensitivity, and orientation-specific calibration factors for all MOSFETs were between $0.63 \%$ and $2.7 \%$. To achieve the highest level of accuracy with dose readings, it is recommended that a specific calibration factor be used for each MOSFET for each energy used. 
Table 4.1. MOSFET calibration factors measured in a $6 \mathrm{MV}$ beam.

\begin{tabular}{|c|c|c|c|}
\hline MOSFET & $\begin{array}{c}\text { Bubble-up } \\
\text { (cGy/mV) }\end{array}$ & $\begin{array}{c}\text { Bubble-down } \\
\text { (cGy/mV) }\end{array}$ & $\begin{array}{c}\text { Average } \\
\text { (cGy/mV) }\end{array}$ \\
\hline High sensitivity & $0.340 \pm 2.7 \%$ & $0.349 \pm 1.8 \%$ & $0.345 \pm 2.5 \%$ \\
\hline $\begin{array}{c}\text { Standard } \\
\text { Sensitivity }\end{array}$ & $0.890 \pm 2.4 \%$ & $0.908 \pm 2.1 \%$ & $0.901 \pm 2.4 \%$ \\
\hline
\end{tabular}

Table 4.2. MOSFET calibration factors measured in a Cobalt-60 beam.

\begin{tabular}{|c|c|c|c|}
\hline MOSFET & $\begin{array}{c}\text { Bubble-up } \\
\text { (cGy/mV) }\end{array}$ & $\begin{array}{c}\text { Bubble-down } \\
\text { (cGy/mV) }\end{array}$ & $\begin{array}{c}\text { Average } \\
\text { (cGy/mV) }\end{array}$ \\
\hline High sensitivity & $0.339 \pm 1.3 \%$ & $0.351 \pm 1.30 \%$ & $0.345 \pm 0.69 \%$ \\
\hline $\begin{array}{c}\text { Standard } \\
\text { Sensitivity }\end{array}$ & $0.875 \pm 0.73 \%$ & $0.899 \pm 0.63 \%$ & $0.887 \pm 1.2 \%$ \\
\hline
\end{tabular}


The calibration factors measured for individual detectors at various field sizes are shown in Fig.4.3. The high sensitivity MOSFETs appeared to have more of a dependence on field size, therefore four MOSFETs were tested under the same conditions at additional smaller field sizes of $4 \times 4 \mathrm{~cm}^{2}, 6 \times 6 \mathrm{~cm}^{2}$, and $8 \times 8 \mathrm{~cm}^{2}$. Figure 4.4 shows the average calibration factors of all MOSFETs tested at each field size, and the uncertainty shown is the standard deviation of the values. There was a negative correlation between field size and calibration factor, as $r=-$ 0.26 for the standard sensitivity MOSFETs and $r=-0.74$ for the high sensitivity MOSFETs, indicating an overall tendency for the calibration factor to decrease with increasing field size. Based on this work, it is recommended that MOSFETs be tested at several field sizes to identify if any small variations in manufacturing have caused the response to change with field size. It was also found that the presence of radio-opaque markers had no measurable effect on the calibration values.

The results of the MOSFETs monitored for change in calibration factor with accumulated dose are shown in Fig.4.5. In all but one case, the last calibration factor measured was significantly lower than earlier measured values, possibly due to the detectors reaching a saturation point earlier than expected. The correlation between accumulated dose and calibration factor was only -0.105 for the standard sensitivity MOSFETs and -0.273 for the high sensitivity MOSFETs, showing very little linear relation between the two values. An ANOVA statistical test, however, shows that the values measured at the various accumulated dose points differ significantly from each other $(t(11)=1.29, p=0.22)$. It is therefore 
recommended to calibrate MOSFETs several times throughout their lifetime (approximately every $4,000-5,000 \mathrm{mV}$ ) for the highest level of accuracy. 
$10 \times 10 \mathrm{~cm}^{2} \square 20 \times 20 \mathrm{~cm}^{2}$

D $15 \times 15 \mathrm{~cm}^{2} \square 25 \times 25 \mathrm{~cm}^{2}$

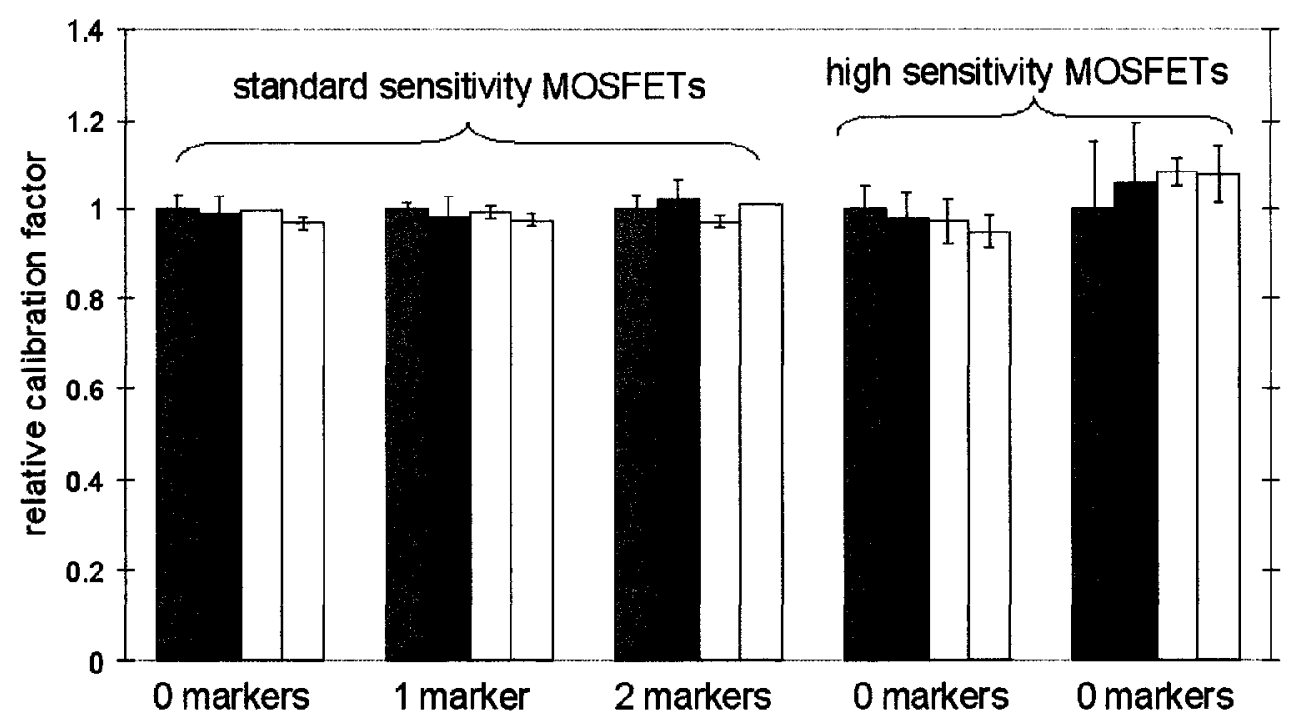

Fig.4.3 Relative MOSFET calibration factors for $10 \times 10 \mathrm{~cm}^{2}, 15 \times 15 \mathrm{~cm}^{2}, 20 \times 20$ $\mathrm{cm}^{2}$ and $25 \times 25 \mathrm{~cm}^{2}$ field sizes for standard sensitivity and high sensitivity MOSFETs normalized to the individual detector response at $10 \times 10 \mathrm{~cm}^{2}$.

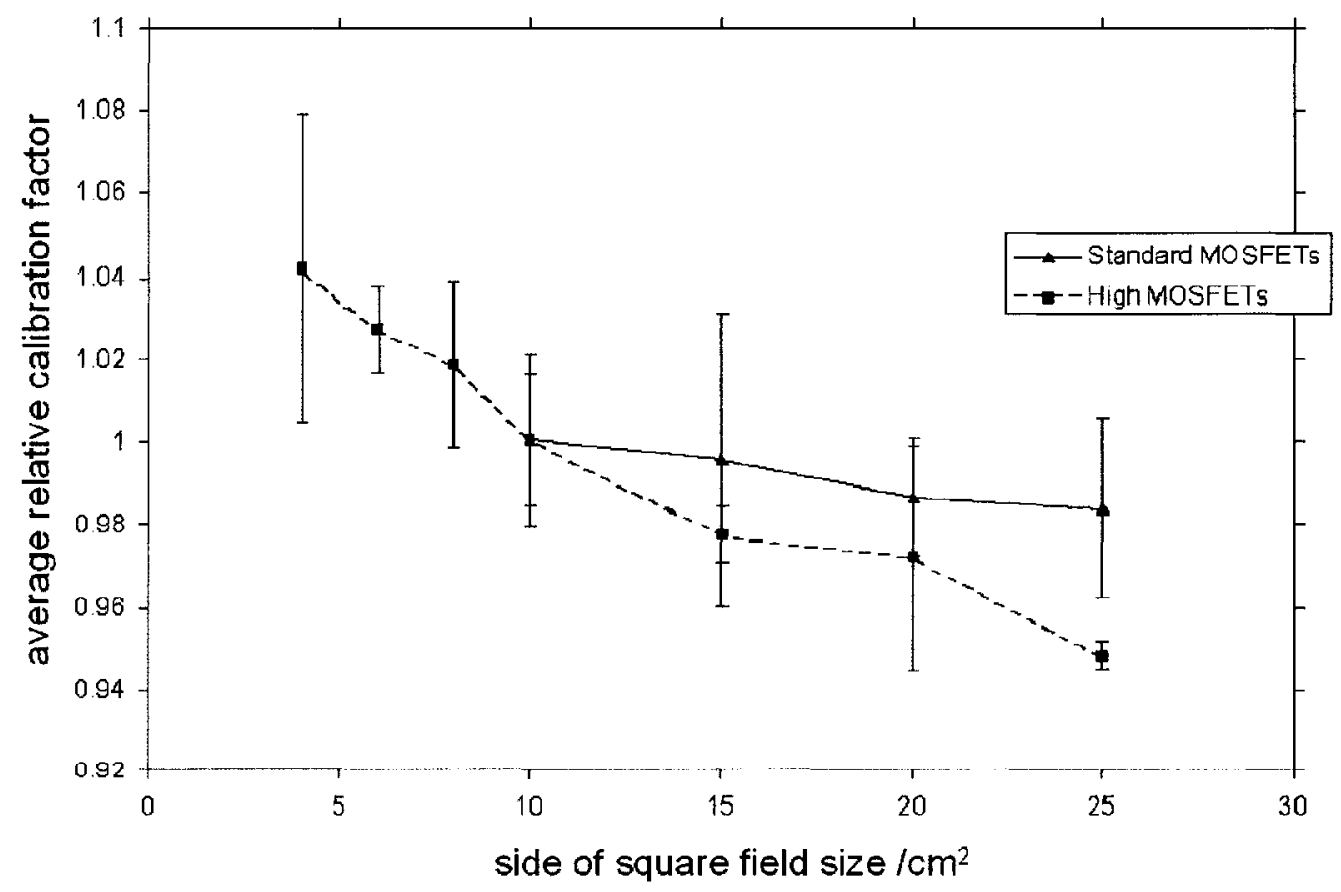

Fig.4.4 Average calibration factors for all MOSFETs tested normalized to the individual detector response at $10 \times 10 \mathrm{~cm}^{2}$, for field sizes $10 \times 10 \mathrm{~cm}^{2}-25 \times 25 \mathrm{~cm}^{2}$ for standard sensitivity and $4 \times 4 \mathrm{~cm}^{2}-25 \times 25 \mathrm{~cm}^{2}$ for high sensitivity. 


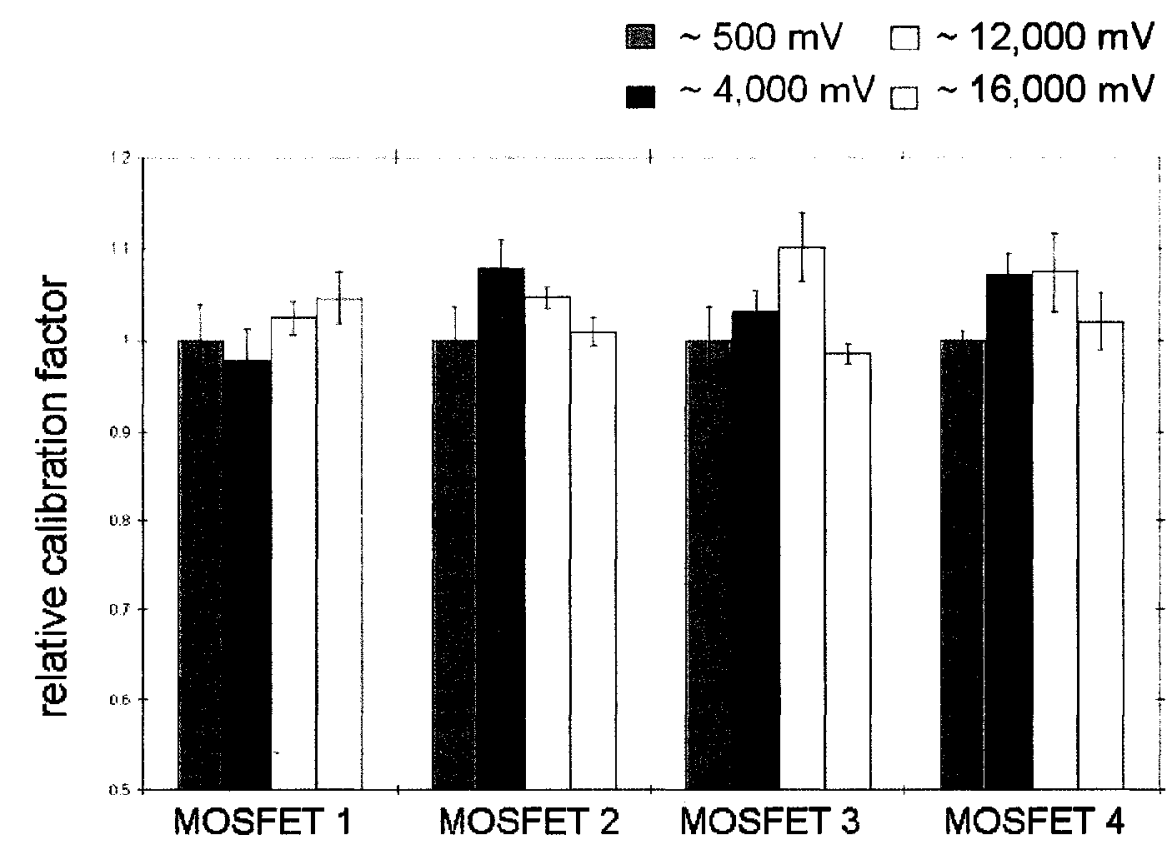

Fig.4.5.a
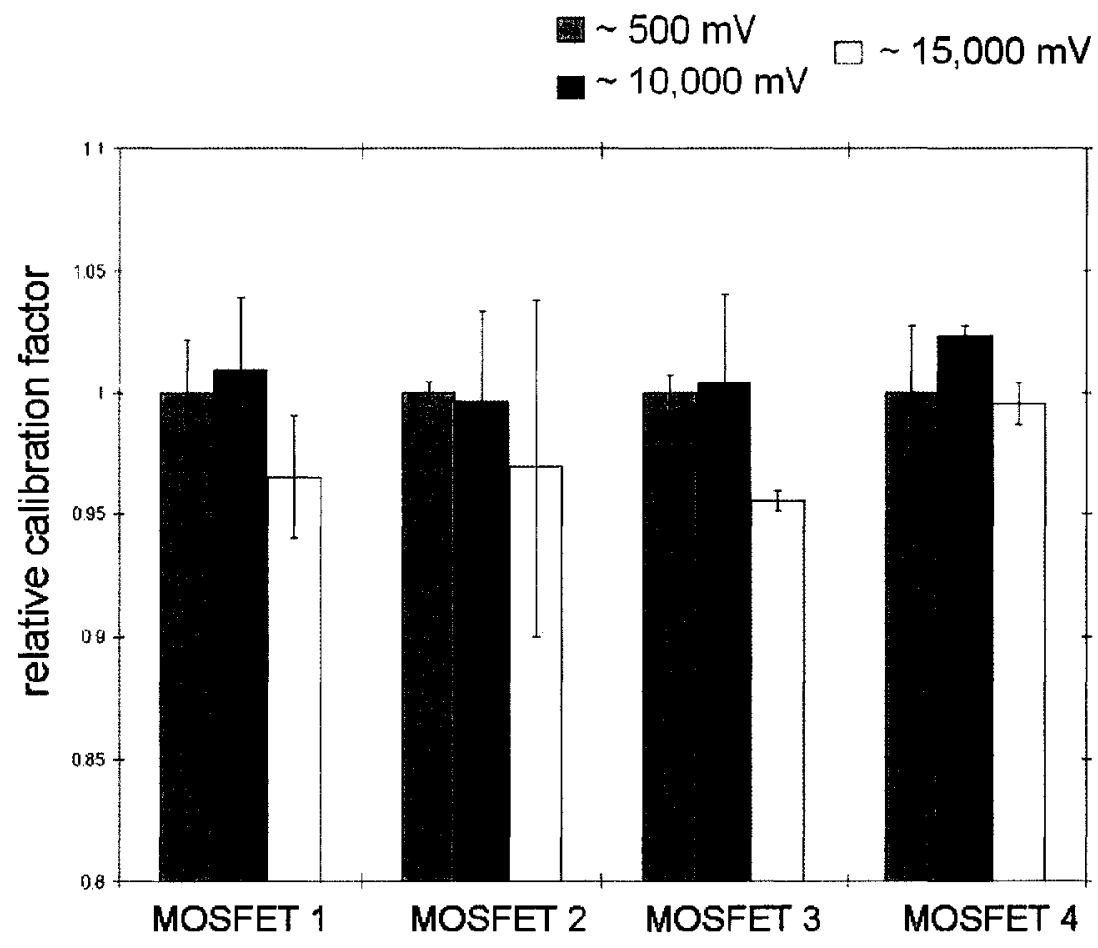

Fig.4.5.b.

Fig.4.5 Relative MOSFET calibration factors for a. standard sensitivity MOSFETs and $b$. high sensitivity MOSFETs measured at various points throughout lifetime of each detector. The legend shows the accumulated voltage of the detectors at the times of calibration. 


\subsection{MOSFET Surface Dose Measurements}

\subsubsection{Procedures}

Once the calibrations were completed, the MOSFETs were tested to determine their accuracy for measuring surface dose in a conventional $6 \mathrm{MV}$ linac. Two calibrated MOSFETs, one standard sensitivity and one high sensitivity, were placed on the surface of a $16.3 \mathrm{~cm}$ slab of solid water approximately $5 \mathrm{~mm}$ from the center.

A Farmer ionization chamber was placed at a depth of $6.3 \mathrm{~cm}$ in the solid water, with $11 \mathrm{~cm}$ as backscatter. The system was set-up at an SSD of $100 \mathrm{~cm}$ and 100 monitor units were delivered three times for each $5 \times 5 \mathrm{~cm}^{2}, 10 \times 10 \mathrm{~cm}^{2}$, and $20 \times 20 \mathrm{~cm}^{2}$ field sizes.

Readings of the threshold voltage of each MOSFET were taken before and after each irradiation. As in the calibration procedure, AAPM's TG-51 protocol was used to determine the dose delivered to the depth of the ionization chamber and percent depth dose curves were then used to calculate the expected dose at the surface, $D_{\text {exp }}(0)$, and at $d_{\max }, D_{\exp }\left(d_{\max }\right)$. The previously determined calibration factors were applied to the change in the threshold voltage of each MOSFET and the resulting dose measurements, $D_{\text {MOSFET }}(0)$, were compared to the expected dose values.

Analysis was done to determine the water equivalent thickness, WED, of each MOSFET tested. This value represents the effective measuring point of the detector. A linear fit was done of a section of the build-up region of the parallel plate ion chamber-measured percent depth dose curve, $P D D_{\text {ionchamber }}$, as seen in 
Fig.4.6. The equation of this fit, Eq.4.5, was then used to extrapolate past the surface.

$$
\begin{gathered}
y=m x+b \\
P D D(d)=m d+P D D(0)
\end{gathered}
$$

The MOSFET-measured percent depth dose at the surface, $P D D_{M O S F E T}(0)$, was determined by calculating the ratio of $D_{\text {MOSFET }}(0)$ to $D_{\text {exp }}\left(d_{\max }\right)$.

$$
P D D_{\text {MOSFET }}(0)=\frac{D_{\text {MOSFET }}(0)}{D_{\text {exp }}\left(d_{\max }\right)} \times 100
$$

The WED was then calculated by solving Eq.3.5 for $d$ using $P D D_{\text {MOSFET }}(0)$ for each MOSFET.

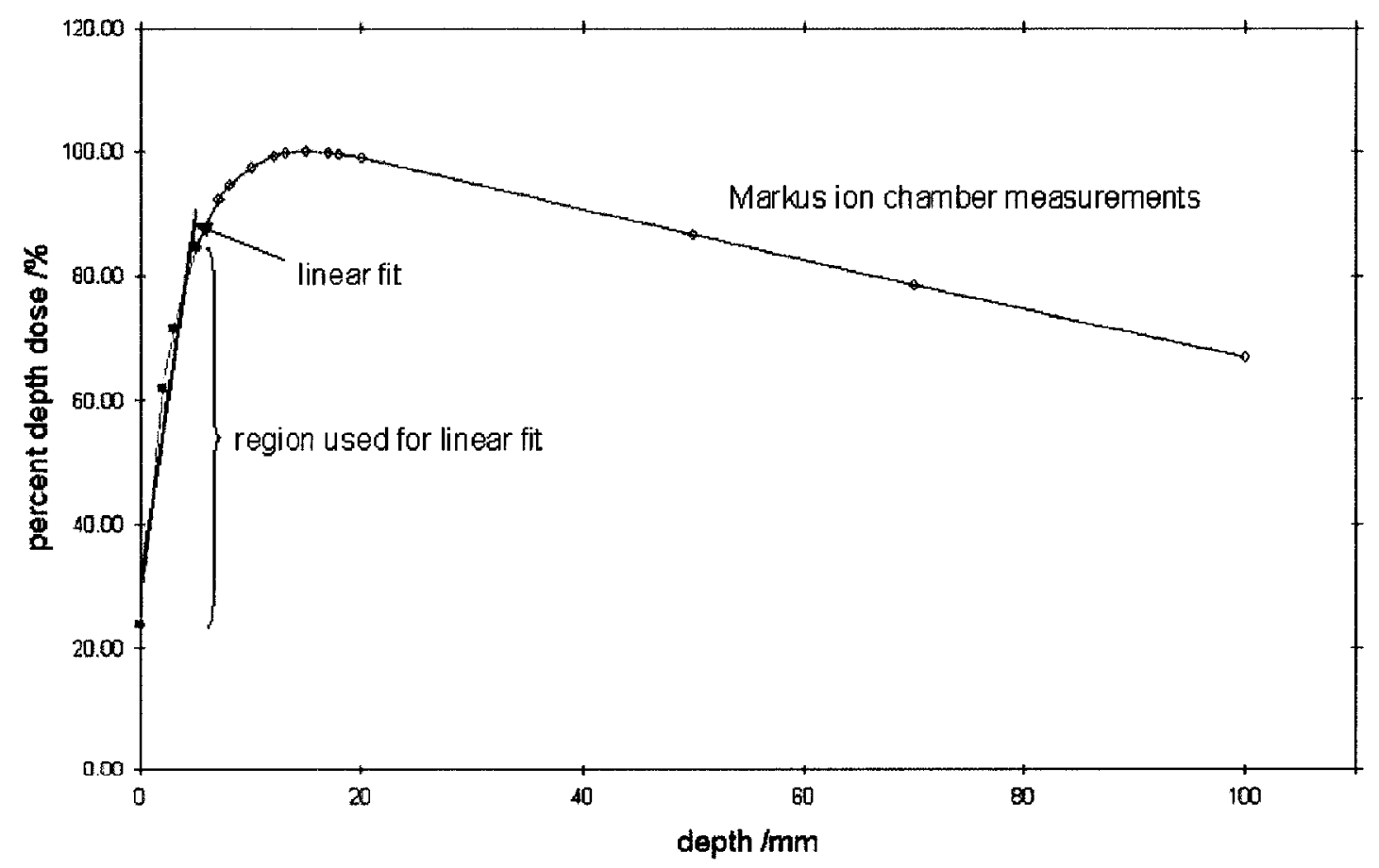

Fig.4.6 Percent-depth dose curve as measured by a Markus ion chamber with a linear fit of the build-up region. 
One high sensitivity MOSFET and one standard sensitivity MOSFET were used to measure percent depth dose curves for a $6 \mathrm{MV}$ linac. One at a time, each MOSFET was embedded bubble-down in the surface of $10.3 \mathrm{~cm}$ of solid water for backscatter, and positioned in the center of a $10 \times 10 \mathrm{~cm}^{2}$ field at an SSD of $100 \mathrm{~cm}$. A dose of $50 \mathrm{cGy}$ was delivered to $d_{\max }$ with the MOSFET at depths varying from $d=0 \mathrm{~cm}$ to $d=10 \mathrm{~cm}$. The MOSFET was irradiated three times at each depth, and the change in threshold voltage was recorded after each trial. The average response at each depth, $\Delta V_{t h}(d)$, was normalized to $\Delta V_{t h}\left(d_{\max }\right)$ to calculate $P D D_{\text {MOSFET }}(d)$ :

$$
P D D_{\text {MOSFET }}(d)=\frac{\Delta V_{t h}(d)}{\Delta V_{t h}\left(d_{\max }\right)} \times 100 \%
$$

Results from both MOSFET trials were compared to $P D D_{\text {ion chamber }}$, which was measured under the same conditions. The water equivalent thickness was also calculated for these detectors using Eq.4.5 where

$$
P D D(d)=P D D_{\text {MOSFET }}(0)=\frac{\Delta V_{t h}(0)}{\Delta V_{t h}\left(d_{\max }\right)} \times 100 \% .
$$

MOSFET measurements were also compared to PDD measurements for the same field size made a high quality diode in a water tank.

\subsubsection{Results}

The deviation of our MOSFET measured surface doses from the expected surface dose is shown in Fig.4.7. The percent difference averaged over all three 
trials and field sizes was $1.55 \%$ for the high sensitivity MOSFET and $5.23 \%$ for the standard sensitivity MOSFET.

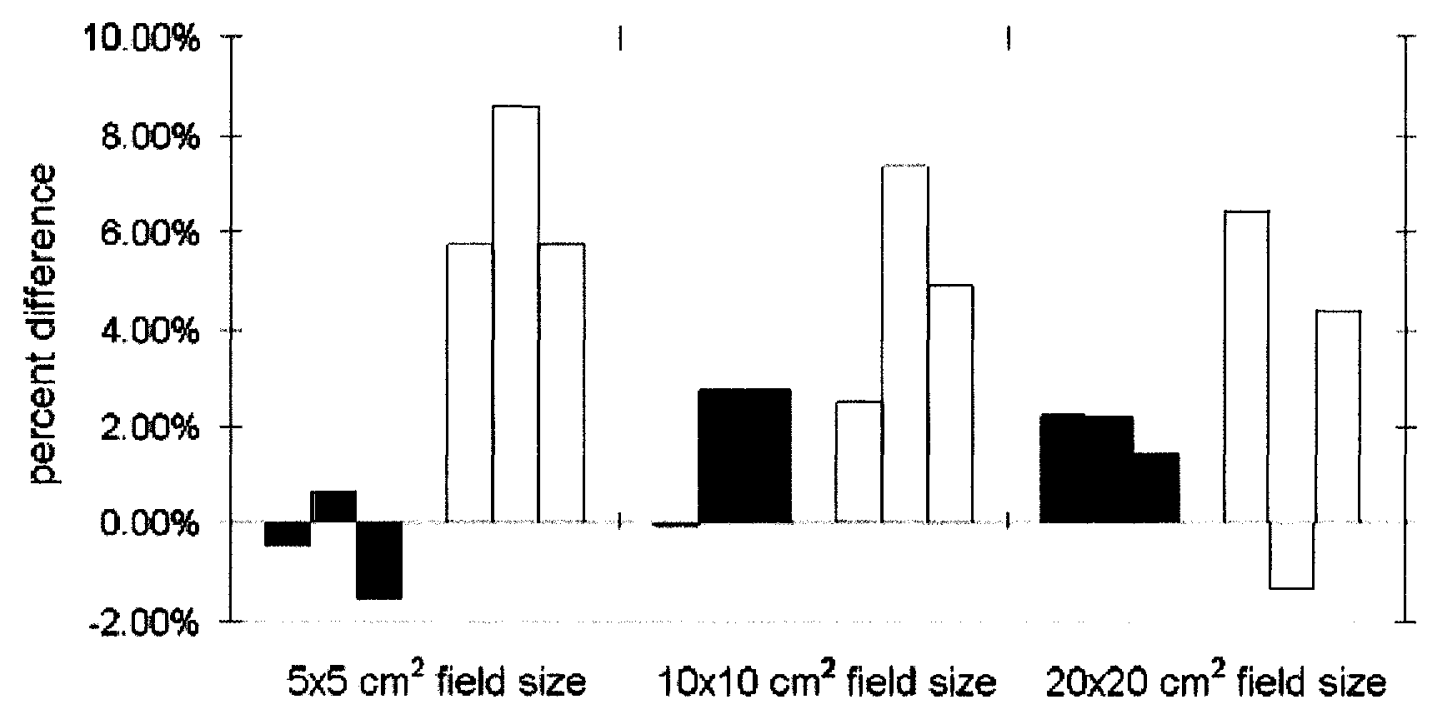

- High sensitivity MOSFET

$\square$ Standard sensitivity MOSFET

Fig.4.7 Comparison of a. standard sensitivity MOSFET and b. high sensitivity MOSFET surface dose readings and the expected surface dose, as calculated from ion chamber readings. 
The average WED obtained for the MOSFETs in both the surface dose experiment and the PDD experiment was $0.73 \pm 0.17 \mathrm{~mm}$ for the high sensitivity detectors and $0.53 \pm 0.25 \mathrm{~mm}$ for the standard sensitivity detectors. The detector-specific WEDs were used to shift the depth values of the $P D D_{\text {MOSFET }}(d)$ measurements downstream, as shown in Fig.4.8 which significantly improved the agreement between the MOSFET data and the ion chamber data.

$$
\left[P D D_{\text {MOSFET }}(d)\right]_{\text {Corr }}=P D D_{\text {MOSFET }}(d+W E D)
$$

The shifted MOSFET data and the ion chamber data can be seen in Fig. 4.9. Since the points were not at the same depth after the shift, linear interpolation was done to compare the results. Averaged over the entire curve, the agreement between the ion chamber measurements and the shifted MOSFET data was $1.1 \%$ for the high sensitivity and $2.6 \%$ for the standard sensitivity MOSFET. Shifting the MOSFET curves by the WED made the greatest improvement in the build-up region, from $d=0 \mathrm{~cm}$ to $d=1.5 \mathrm{~cm}$, as the agreement improved from $9.82 \%$ to $0.97 \%$ for the high sensitivity MOSFET and from $6.12 \%$ to $3.30 \%$ for the standard sensitivity MOSFET in this region. Similar agreement was also found when the MOSFET data was compared with diode measurements. 


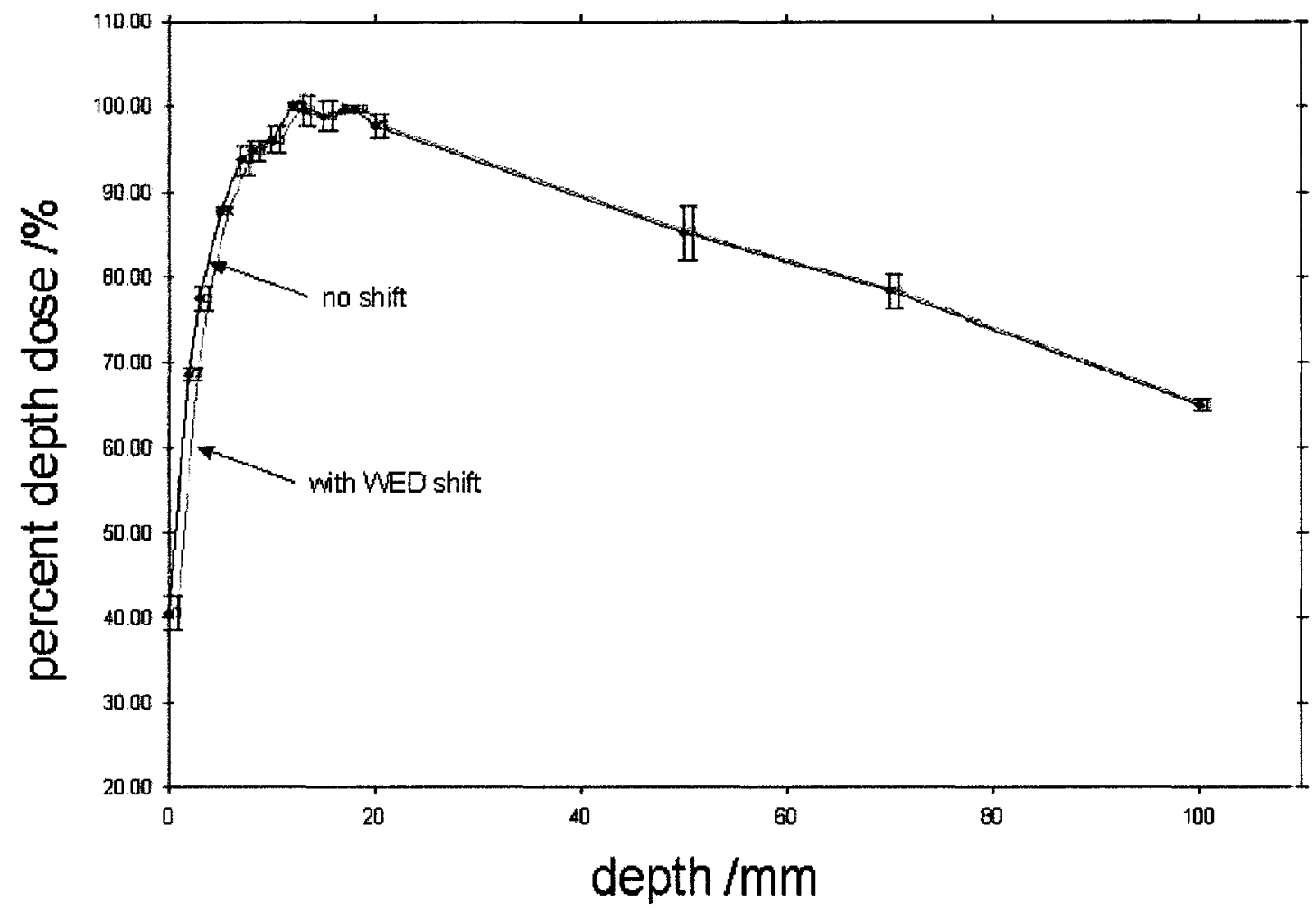

Fig.4.8 Percent depth dose curve measured with a high sensitivity MOSFET. Data is presented both with and without shift of water equivalent depth. 


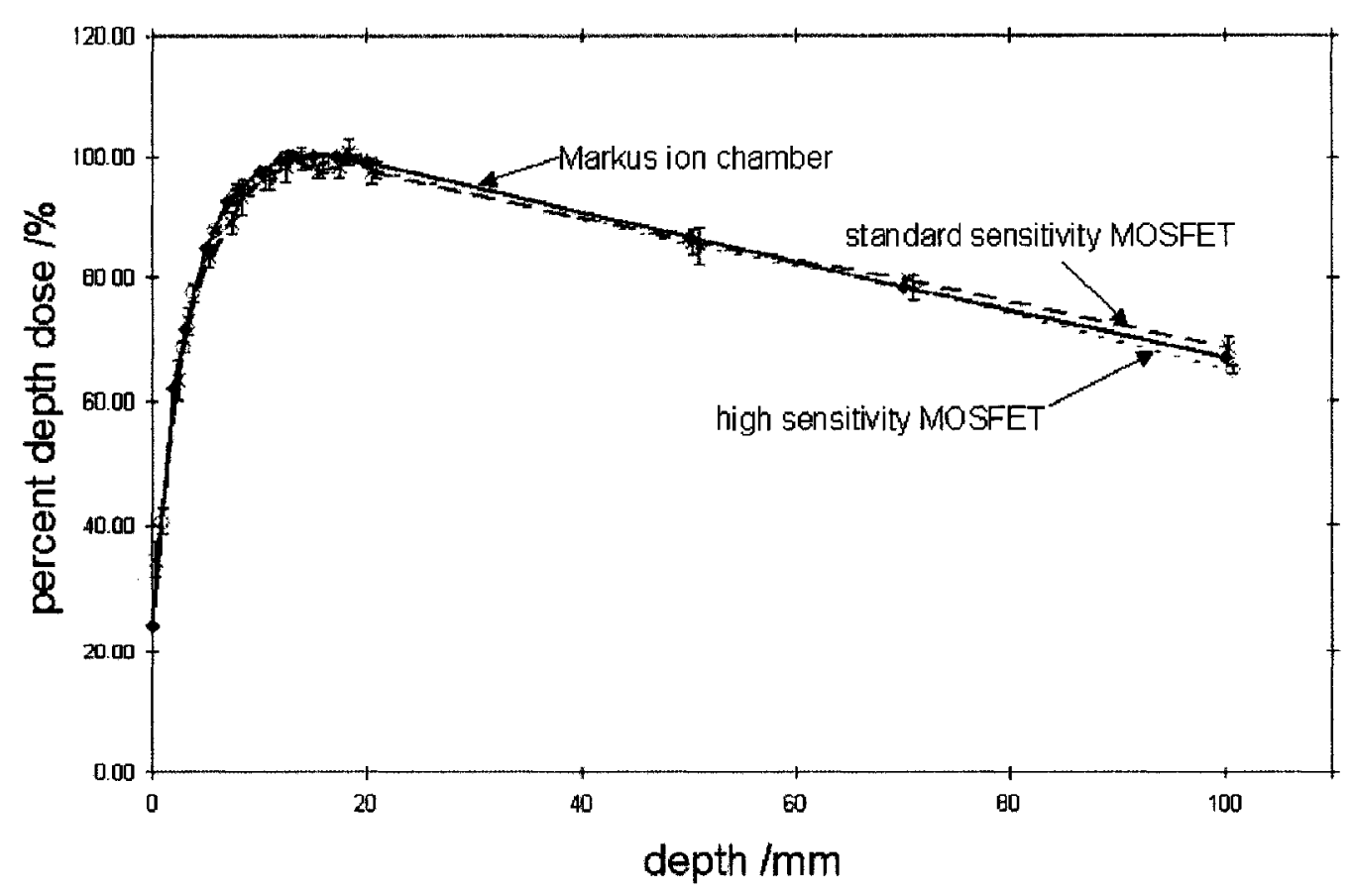

Fig.4.9.a.

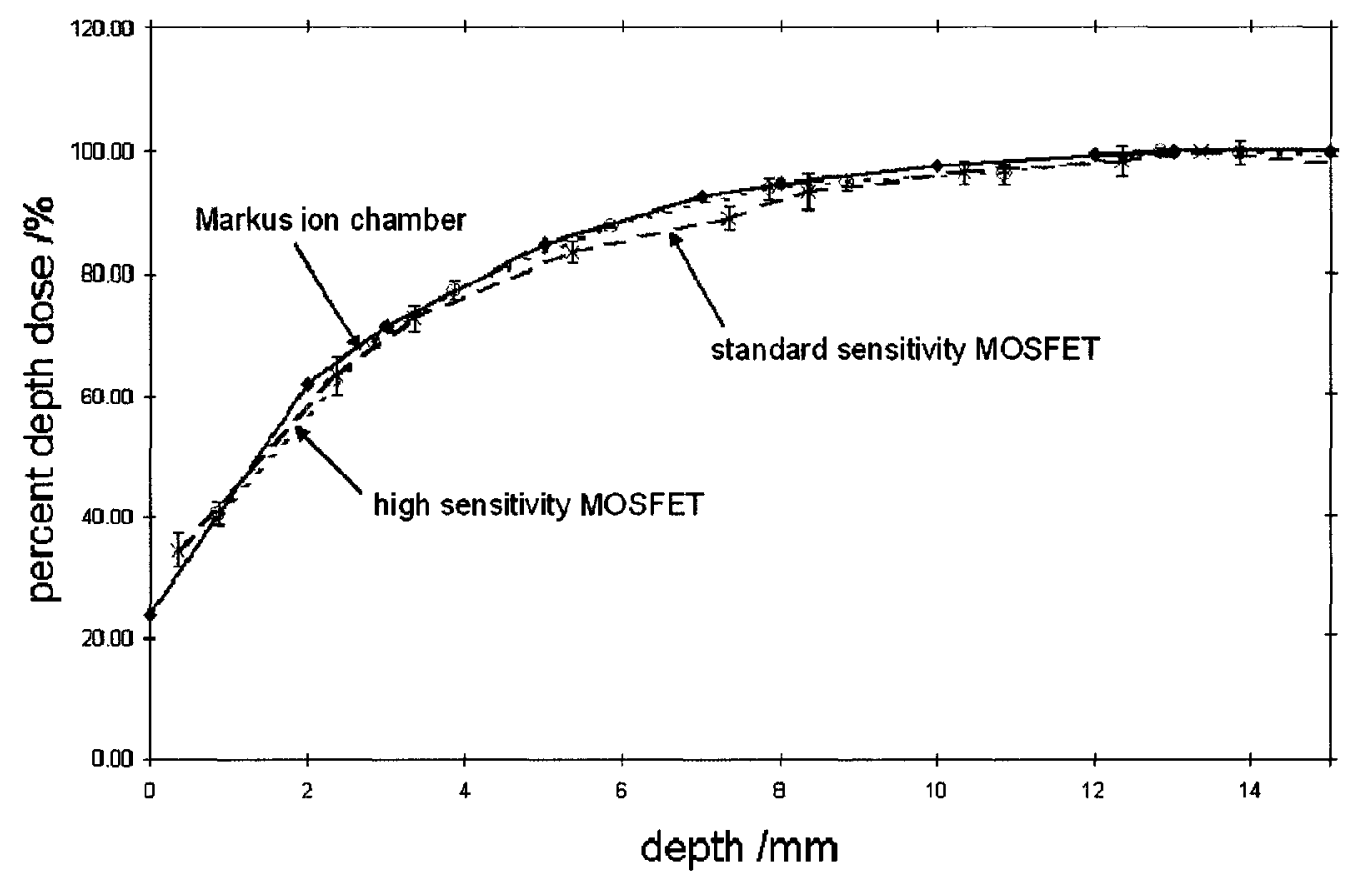

Fig.4.9.b.

Fig.4.9 Comparison of percent depth dose curves as measured by a standard sensitivity MOSFET, a high sensitivity MOSFET and a Markus ion chamber. a. entire curve and $b$. build-up region. 


\section{Chapter 5}

\section{In vivo Measurements}

\section{1 "In vivo" Measurements with Anthropomorphic Rando Phantom}

Small positioning errors in high dose gradients can lead to large errors in dose measurements. Therefore, before any measurements were made on patients, a Tomotherapy head and neck treatment was simulated on an anthropomorphic RANDO phantom to evaluate such errors. This allowed for consecutive irradiations and measurements without moving the phantom. The purpose of this study was to evaluate the reproducibility of MOSFET measurements during a Tomotherapy treatment with minimal error due to patient positioning uncertainties.

\subsubsection{Treatment Preparation}

An S-frame immobilization mask was made for the head and neck portion of the RANDO phantom and the mask was marked with reference points to indicate the positions of MOSFETs for future measurements. Ball bearings (bb's) that are visible in CT images were placed as the fiducial markers at the reference points on the mask, see Fig.5.1. The RANDO phantom and mask were then positioned on the couch of a Philips Brilliance Computed Tomography scanner (Koninklijke Philips Electronics N.V., Netherlands). 


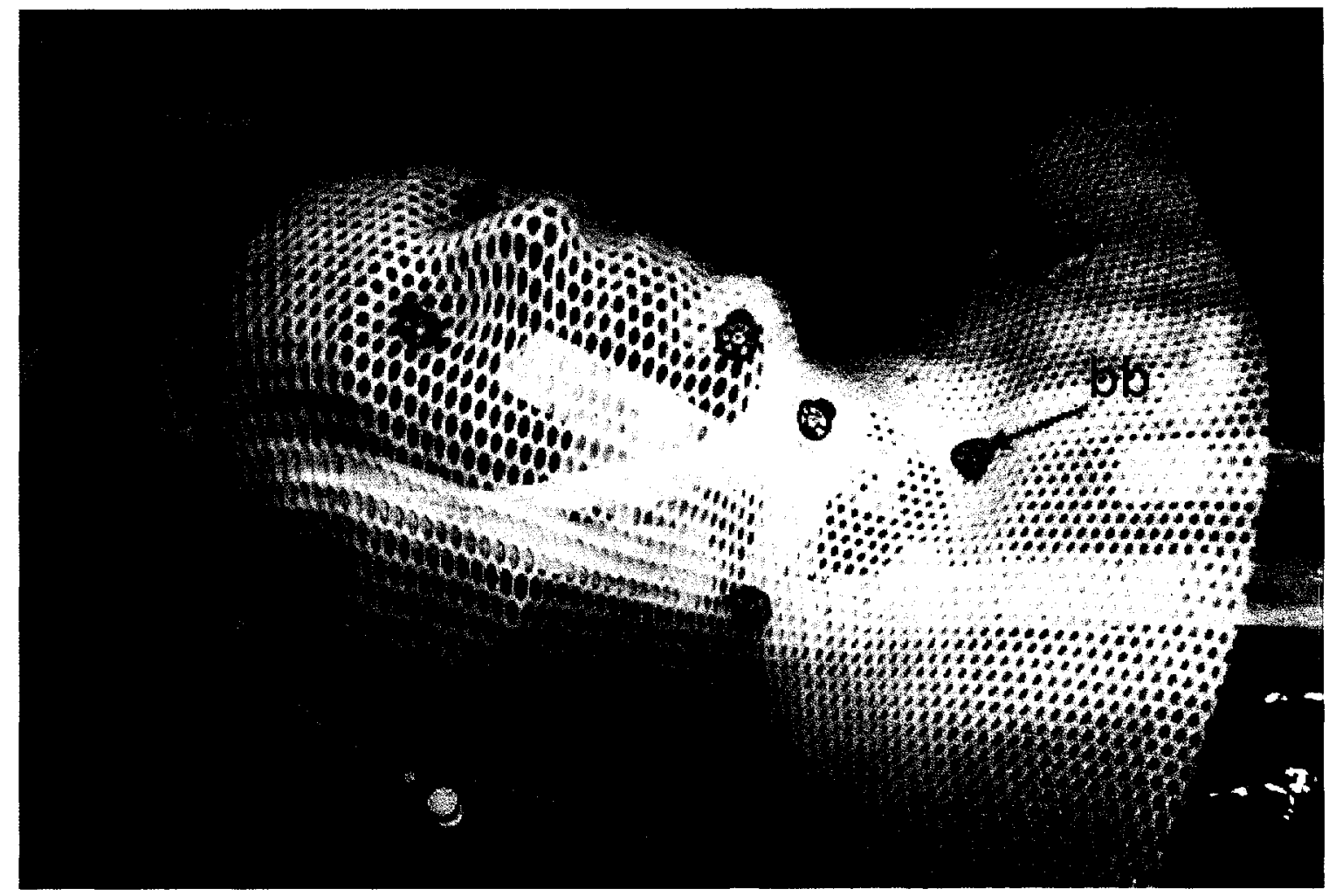

Fig.5.1 Anthropomorphic RANDO phantom and S-frame immobilization mask, with points of interest marked with bb's. 
The phantom was then scanned and the images were exported to a Pinnacle treatment planning system (Koninklijke Philips Electronics N.V., Netherlands). In Pinnacle, contours of organs at risk, OARs, as well as target volumes were created for a typical laryngeal cancer treatment plan (Table 5.1)

Table 5.1 List of organs at risk and target volumes contoured for treatment plan.

\begin{tabular}{|c|c|}
\hline Organs at risk: & $\begin{array}{l}\text { Spinal cord } \\
\text { Brain stem } \\
\text { Right lung, left lung } \\
\text { Right parotid gland, left parotid gland } \\
\text { Right eye, left eye } \\
\text { External } \\
\text { Skin sparing structure }\end{array}$ \\
\hline Target Volumes: & $\begin{array}{l}\text { Gross Tumor Volume, GTV } \\
\text { Clinical Target Volume, CTV } \\
\text { Planning Target Volume, PTV }\end{array}$ \\
\hline
\end{tabular}


Between the PTV and the external contour, a small skin-sparing structure was created as seen in Fig.5.2. The image set and contours were then sent to the Tomotherapy treatment planning system where two different treatment plans were created. The plans were both done on a fine grid, with $3 \mathrm{~mm}$ thick slices and $2.34 \times 2.34 \mathrm{~mm}^{2}$ pixels. Plan 1 was designed to deliver typical doses to the PTV, sparing OARs, but with no importance (zero weighting) given to the sparing of the skin structure. A second plan, Plan 2, was designed in the same way, but with an effort to spare the skin structure, in the area where a high skin dose was expected due to the proximity of the target to the phantom surface. This method was evaluated with both MOSFET dosimetry and the treatment planning system for its effectiveness in lowering skin dose. This procedure has been shown in literature to reduce skin dose by an average of $(23.0 \pm 6.8) \%$ for treatments using IMRT with a linac [5]. 


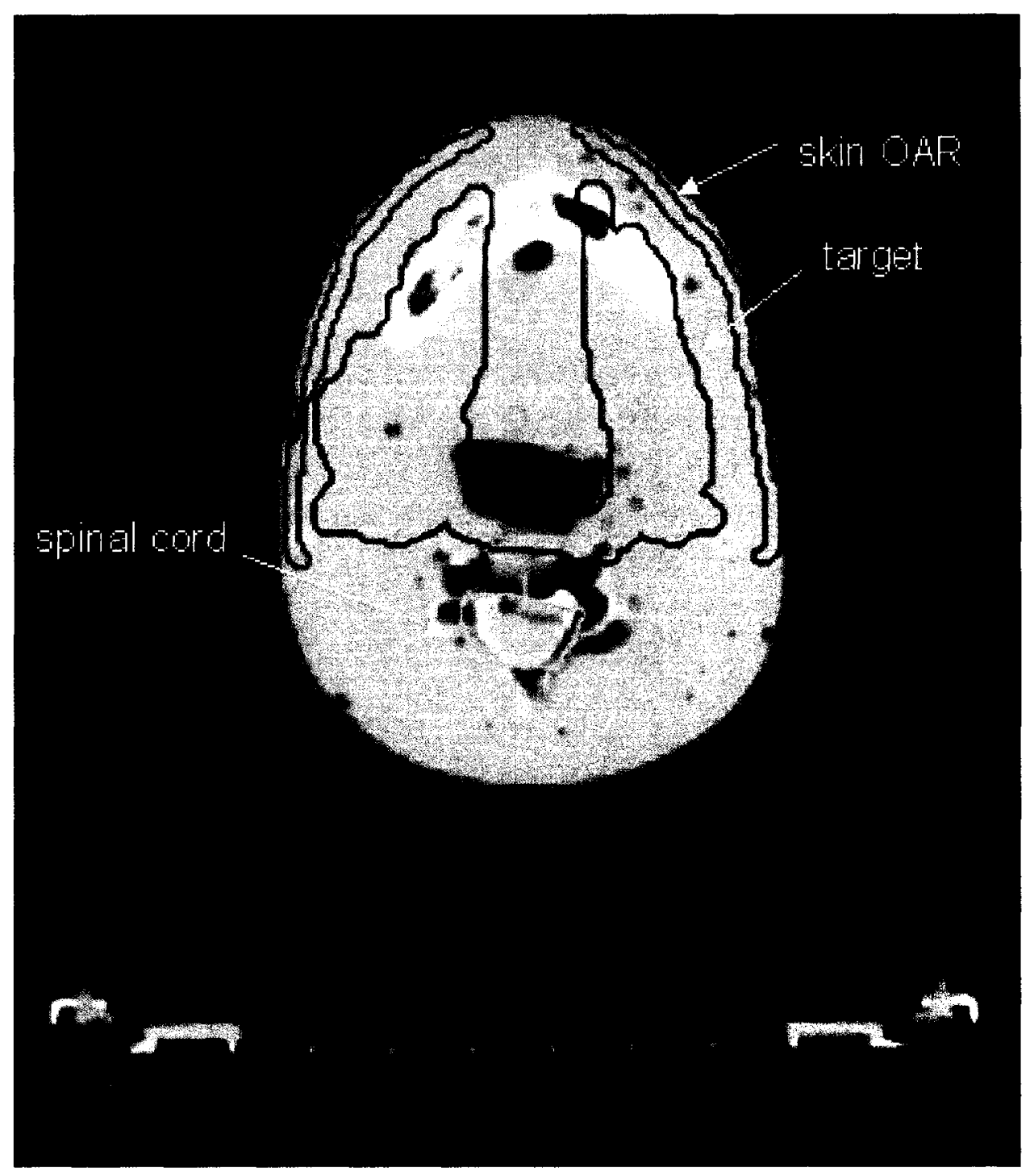

Fig.5.2 Screen capture of the treatment plan showing contours of the spinal cord, target, and skin sparing structure. 


\subsubsection{Reproducibility Measurements}

"In vivo" measurements were made on our RANDO phantom to evaluate the reproducibility and accuracy of MOSFETs during treatment on a Tomotherapy HiArt unit. The phantom was placed in the supine position on the treatment couch, and the immobilization mask was attached. Five MOSFETs, which were connected to the standard bias supply and reader, were taped bubble-down on top of the immobilization mask at the reference points. Both high and standard sensitivity MOSFETs were used for these measurements.

The phantom was then scanned using the Megavoltage CT scanner included in the Tomotherapy unit. The acquired MVCT images were then registered with the original kilovoltage CT images that were used for the treatment planning, Fig. 5.3. Image registration is a powerful tool as it allows the user to adjust the MVCT images to adequately match the original kVCT. This process identifies any adjustments that should be made to the patient's position to account for shifts of internal organs from day to day as well as to correct positioning errors.

Once the couch was adjusted accordingly, an initial reading of the threshold voltage of the detectors was taken. Radiation treatment was then delivered to the phantom using Plan 1, which had no skin-sparing considerations. The MOSFETs were read after the irradiation and calibration factors were applied to obtain the dose at each point. Without moving the phantom, the mask and MOSFETs were removed and then replaced on the reference marks before the phantom was irradiated again. A reading of the threshold voltages was taken before and after the treatment. This entire procedure was repeated three times a day on three 
different days, and each time care was taken to assure proper alignment of the phantom and accurate positioning of the MOSFETs. Measurements were also taken with TLD chips at the same reference points for comparison. The bb's that were imaged on the immobilization mask during the original CT scan were used to determine the dose at the reference points underneath and on top of the mask as calculated by the Tomotherapy treatment planning system, Fig. 5.4.

a.

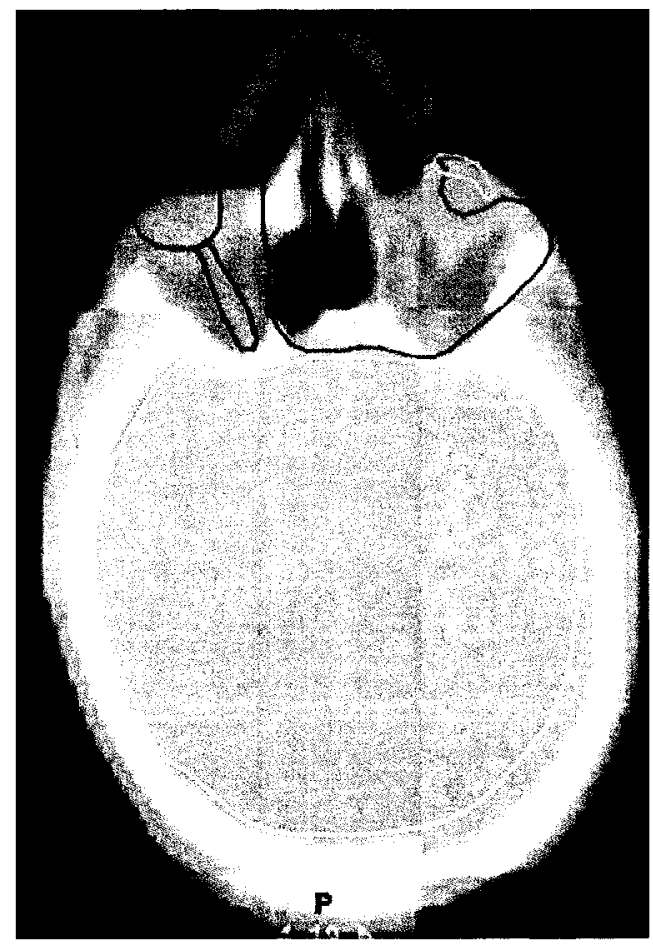

b.

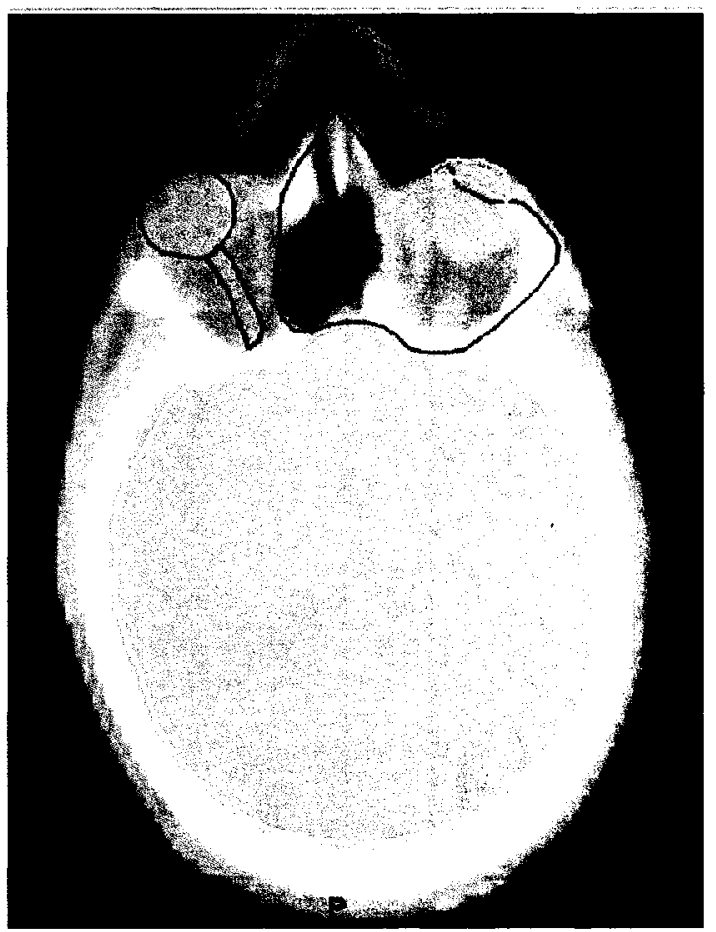

Fig.5.3 Tomotherapy image registration showing the daily MVCT (grey image) and the original KVCT used for treatment planning (yellow image) a. before and b. after shifts. 


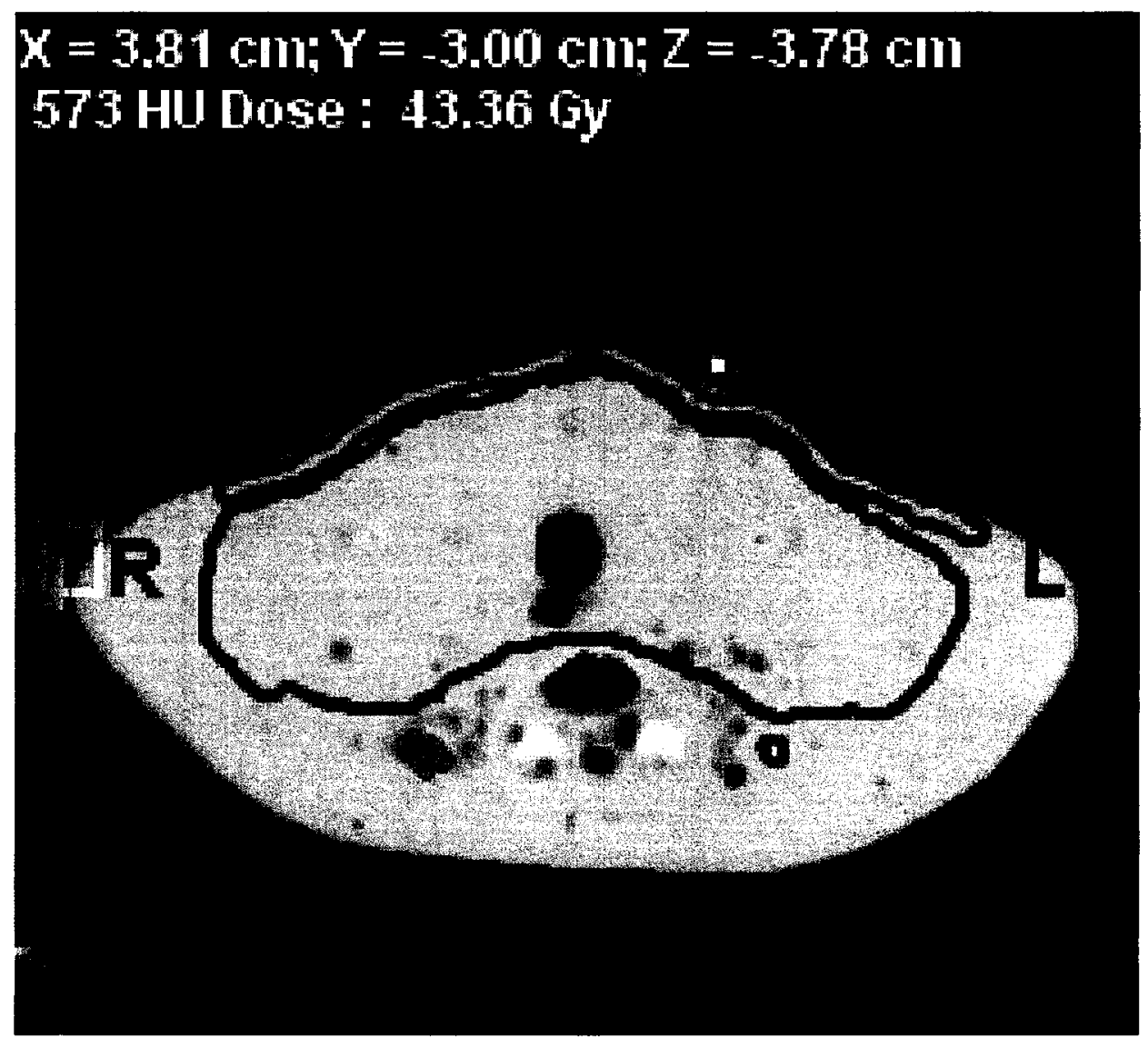

Fig.5.4 Screen capture of the treatment plan showing the position of the bb and the Hounsfield unit and the total dose assigned to the voxel. 


\subsubsection{Evaluation of Skin as Organ at Risk}

The RANDO phantom was also treated on the Tomotherapy unit to evaluate the effect of skin sparing in Plan 2, which considered the skin an OAR, with respect to the skin doses delivered by Plan 1, which had no skin sparing considerations. The phantom was set-up on the treatment couch as described before, and the MOSFETs were positioned directly on the phantom surface, underneath the S-frame mask. After the phantom was scanned and properly positioned using image registration, initial readings of the MOSFETs were taken. The phantom was then treated with Plan 1 and then Plan 2 consecutively. This was repeated three times. The MOSFETs were read after each irradiation and neither the detectors nor the phantom was moved throughout the entire procedure to ensure consistent positioning.

\subsubsection{Results}

\section{Reproducibility}

Table 5.1 shows the doses measured by the MOSFETs on our RANDO phantom, averaged over a total of nine trials. The inter-fraction deviation of the MOSFET measurements, which can be seen in Fig.5.5, was between $2.2 \%$ and $3.8 \%$ of the average dose at each specific location. This was within the expected range as each individual dose measurement had an uncertainty of approximately $3 \%$ due to the standard deviation of the calibration factors used and reproducibility of the system. Additional uncertainty due to removing and replacing the MOSFETs between each trial was also expected. Agreement with TLD measured doses was generally good, as the difference ranged from $2.2 \%$ to 
$-6.0 \%$, with an absolute average of $(4.1 \pm 1.8) \%$. The treatment plan doses are shown in Table 5.2 as an average of the dose values assigned to all voxels which contained a part of the bb or MOSFET marker each day and the uncertainty is the standard deviation of these values. The difference between the MOSFET measured dose and the calculated treatment plan dose at each point was as high as $13.2 \%$, with an absolute average of $(7.8 \pm 4.4) \%$. This large deviation was investigated by reviewing the skin dose calculations done by the Tomotherapy treatment planning system, as later described in Section 5.2.2. 
Table 5.2 In vivo dose measurements and Tomotherapy treatment plan dose values for RANDO phantom.

\begin{tabular}{|l|c|c|c|c|c|}
\hline \multirow{2}{*}{ Position } & $\begin{array}{c}\text { MOSFET Dose } \\
\text { (OGy) }\end{array}$ & \multicolumn{2}{|c|}{ TLD } & \multicolumn{2}{c|}{ Treatment Plan } \\
\cline { 3 - 6 } & & Dose (OGy) & $\%$ Diff from MOSFET & Dose (CGy) & $\%$ Diff from MOSFET \\
\hline 1 (under mask) & $158.2+3.8 \%$ & $165.4+2.2 \%$ & $4.4 \%$ & $145.6+2.3 \%$ & $.83 \%$ \\
\hline 2 (under mask) & $176.4+2.2 \%$ & $180.3+4.3 \%$ & $2.2 \%$ & $174.7+5.7 \%$ & $-1.0 \%$ \\
\hline $\begin{array}{l}3 \text { (on top of } \\
\text { mask) }\end{array}$ & $144.3+2.7 \%$ & $152.4+1.9 \%$ & $5.4 \%$ & $157.2+3.0 \%$ & $8.6 \%$ \\
\hline $\begin{array}{l}4 \text { (on top of } \\
\text { mask) }\end{array}$ & $159.3+2.6 \%$ & $163.1+5.2 \%$ & $2.4 \%$ & $173.0+1.5 \%$ & $8.2 \%$ \\
\hline $\begin{array}{l}5 \text { (on top of } \\
\text { mask) }\end{array}$ & $149.2+2.9 \%$ & $140.6+3.9 \%$ & $.60 \%$ & $170.2+1.2 \%$ & $13.2 \%$ \\
\hline
\end{tabular}




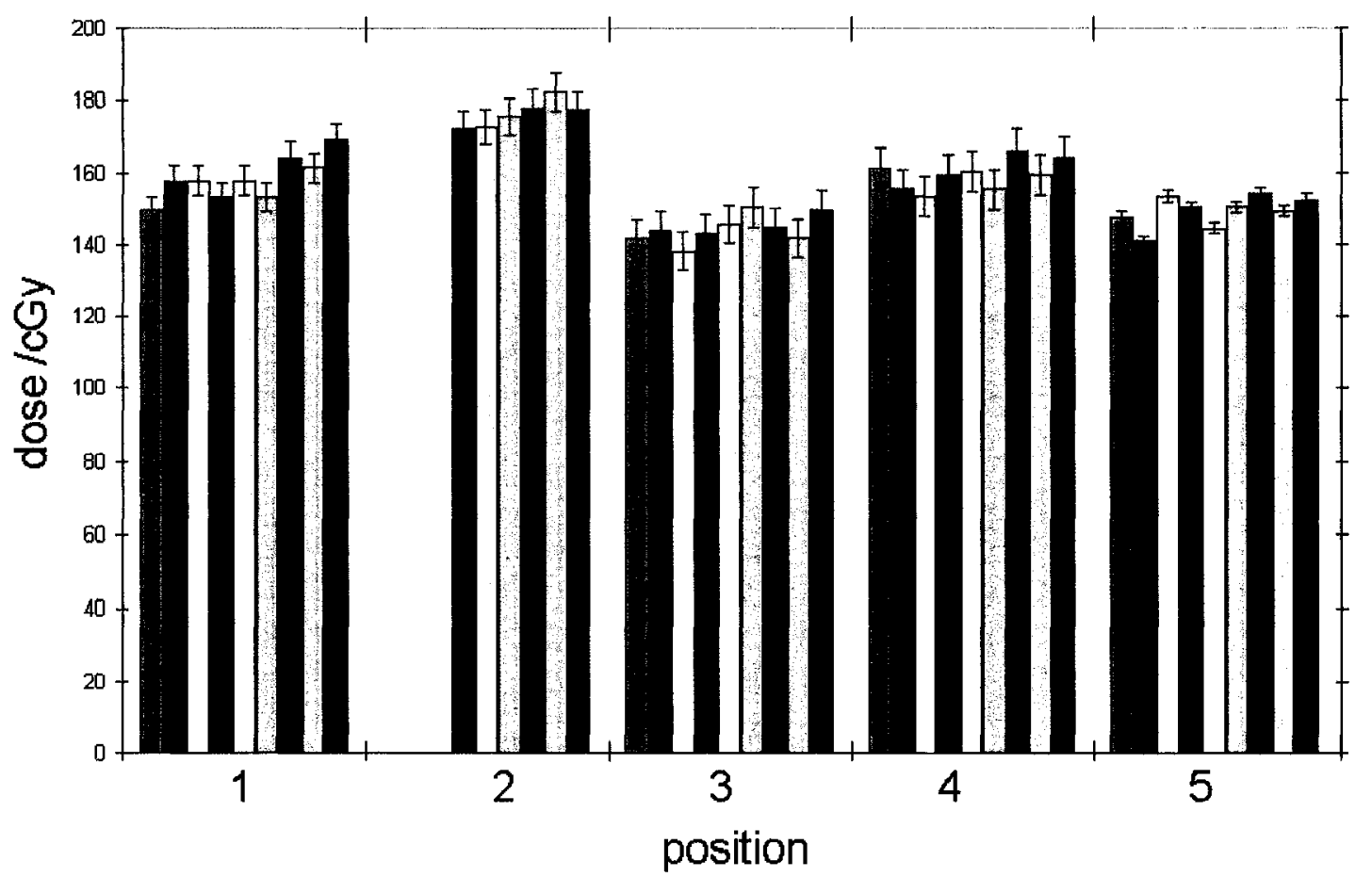

Fig.5.5 MOSFET dose measurements taken on the RANDO phantom at five points of interest. The first three measurements at position 2 are absent due to a malfunction of the MOSFET during the experiment. The uncertainty shown is from the standard deviation of the original calibration factors. 


\section{Evaluation of Skin as Organ at Risk}

The doses measured by the MOSFETs for both the non-skin sparing plan, Plan 1, the skin-sparing plan, Plan 2, are listed in Table 5.3. The average reduction in dose was $(19.7 \pm 6.3) \%$, which is consistent with previous findings for IMRT with a linac (27). As predicted by the treatment planning system, there was a significant reduction in skin dose when the skin-sparing plan, Plan 2, was used, as seen in Figs. $5.6 \mathrm{a}$ and $5.6 \mathrm{~b}$. The volume of the skin sparing structure that received more than 45 Gy was reduced from $95 \%$ to $60 \%$. The isodose curves showed that this was achieved during optimization of the plan by having some of the beams enter through the back of the phantom. At the same time the dosevolume histograms indicate that the main organ at risk in this region, the spinal cord, did not receive a significant amount of increased dose. Having the beams enter the body around the cord, rather than directly through it, spared this OAR. The MOSFET detectors were ideal for these measurements since they could be left in place throughout the entire experiment, ranging over all treatment fractions. This eliminated any error due to misplacement and ensured that the dose was being measured at the same location for each treatment. 
Table 5.3 MOSFET measured doses for treatment of RANDO phantom with Plan 1, with no skin sparing, and Plan 2, with skin sparing.

\begin{tabular}{|c|c|c|c|c|}
\hline Position & Plan 1 (cGy) & Plan 2 (cGy) & $\begin{array}{c}\text { Dose (cGy) } \\
\text { (Plan 1-Plan 2) }\end{array}$ & \% Difference \\
\hline 1 & $175.5 \pm 5.7$ & $155.8 \pm 4.4$ & 19.6 & $11.9 \%$ \\
\hline 2 & $184.8 \pm 1.9$ & $149.1 \pm 2.6$ & 35.7 & $21.4 \%$ \\
\hline 3 & $1873 \pm 0.8$ & $161.2 \pm 6.6$ & 26.1 & $15.0 \%$ \\
\hline 4 & $172.5 \pm 2.5$ & $137.4 \pm 2.5$ & 35.0 & $22.6 \%$ \\
\hline 5 & $184.6 \pm 1.5$ & $139.7 \pm 4.7$ & 44.9 & $27.7 \%$ \\
\hline
\end{tabular}




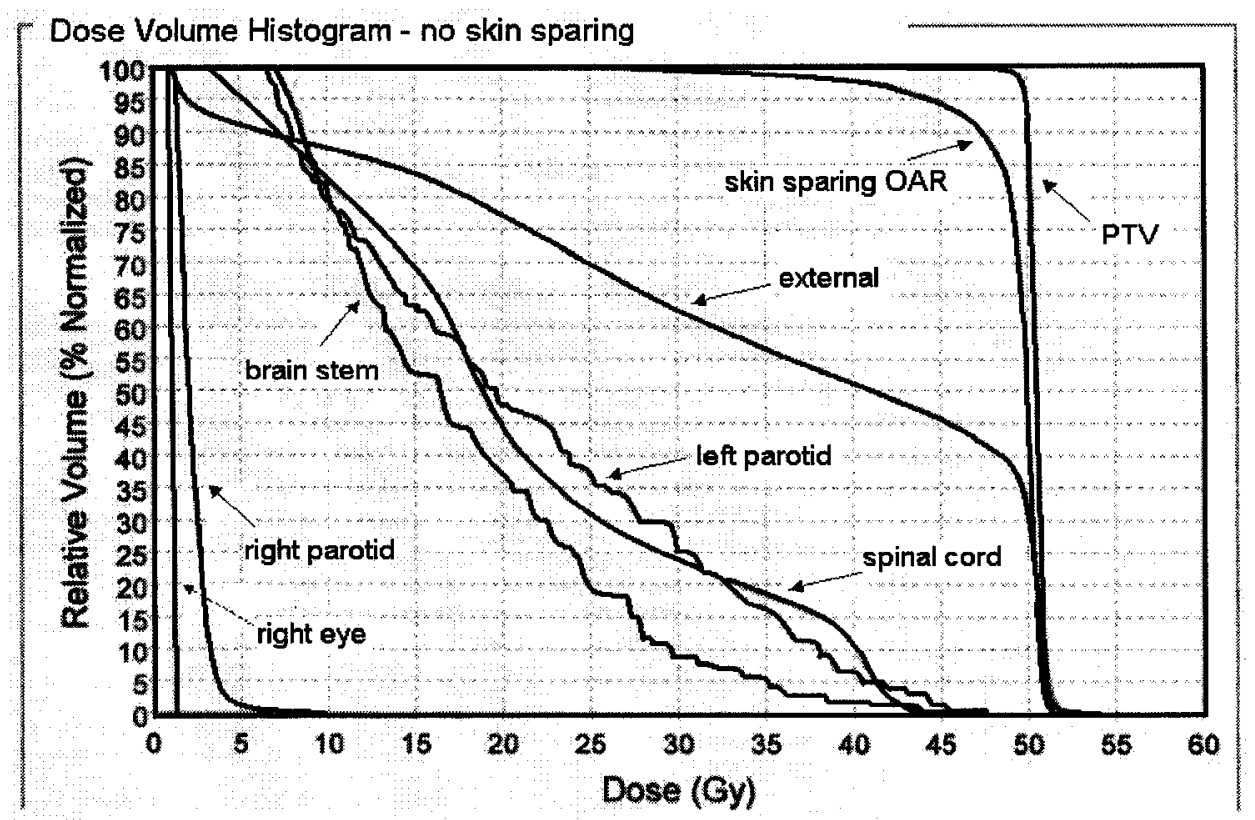

Fig.5.6.a.

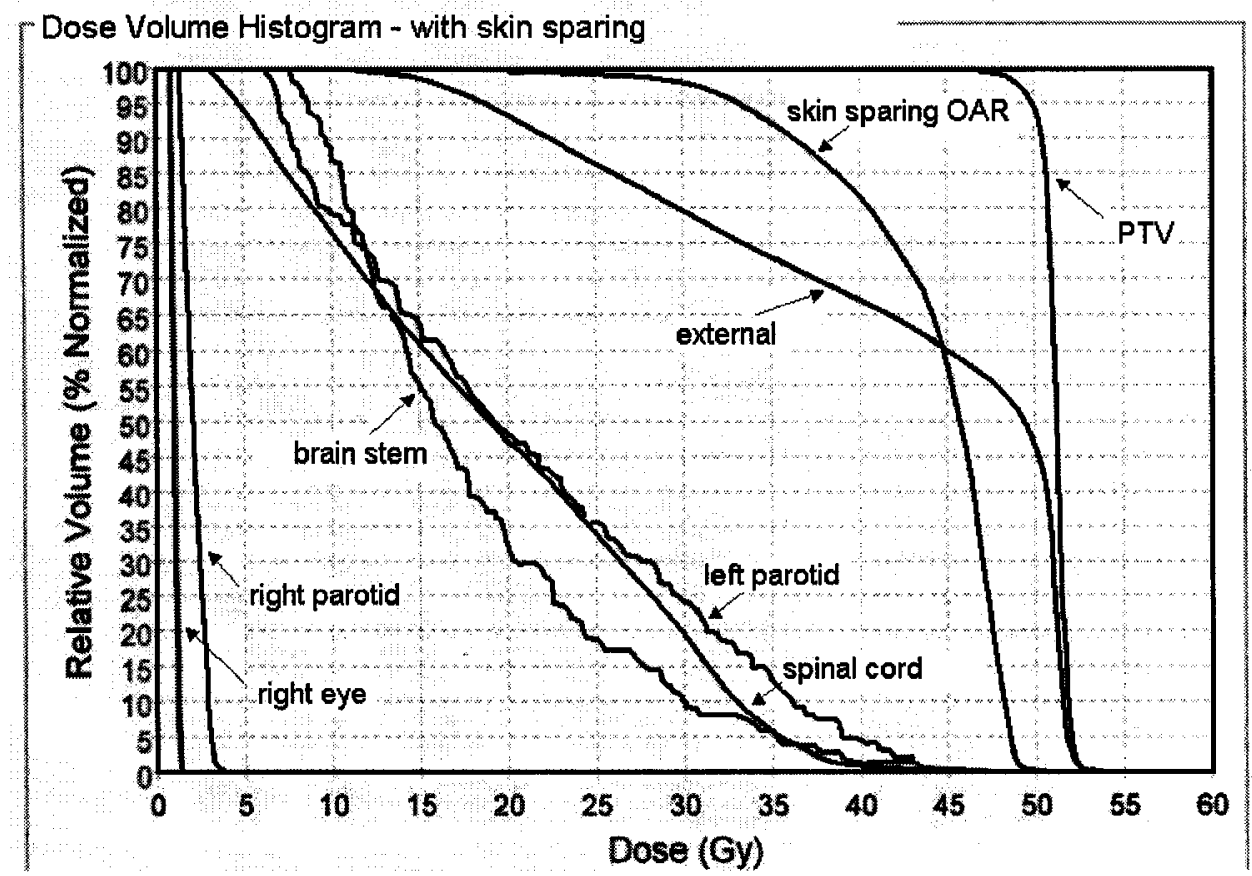

Fig.5.6.b.

Fig.5.6 Dose-volume histograms generated from a. Plan 1, with no effort to spare the skin OAR and b. Plan 2, with sparing of the skin OAR. 


\subsection{In vivo Head and Neck Patient Measurements}

The purpose of this study was to evaluate the accuracy and reproducibility of MOSFET skin dose measurements on patients treated with Tomotherapy. Patient selection was focused on people with cancer of the head and neck due to the high skin dose received during such treatment. The goal was to take ten measurements at the same five reference points throughout the treatment of each patient used in the study. Some patients needed to have a second immobilization mask made after only a couple of treatments due to significant weight loss, which did not allow for consistent measurements at the same points. In this case, the previous measurements were disregarded. This was also the case if the treatment plan itself was altered after only a few measurements. By the end of the study, measurements were completed on five patients.

Measurements were also taken at the same points on each mask using TLDs, and the calculated treatment plan dose was determined using the Tomotherapy software.

\subsubsection{Procedures}

Before the commencement of treatment, each patient's immobilization mask was marked with reference points in regions that were expected to receive a high dose to indicate where the MOSFETs would be placed for daily measurements. The example shown in Fig.5.7 was a treatment plan for a patient with a target to the right of the nose. The area below the right eye received a high dose, therefore the MOSFETS were placed in this region, as can be seen in Fig.5.8. Preliminary trials were done on one patient placing the MOSFETs under the 
mask, in direct contact with skin; however, it was very difficult to accurately reproduce MOSFET positions from day to day. For all patient measurements presented in this work, the MOSFETs were used on top of the mask, as this provided the best opportunity to assure accurate positioning.

On each day of measurements, five MOSFETs were connected to the standard bias supply and reader at least one hour prior to irradiations. Approximately ten minutes before the treatment began, the initial threshold voltages were read and the bias supply and MOSFETs were disconnected from the reader and transported to the treatment room. The MOSFETs were carefully taped on top of the immobilization mask at the reference points in the bubble-down position. The MOSFETs remained in this position throughout the CT scan and treatment. Once the treatment was complete, the MOSFETs were removed from the mask, connected to the reader, and read after a two-minute wait period. Previously determined calibration factors were applied to the readings to obtain the dose delivered to each MOSFET during the irradiation. 


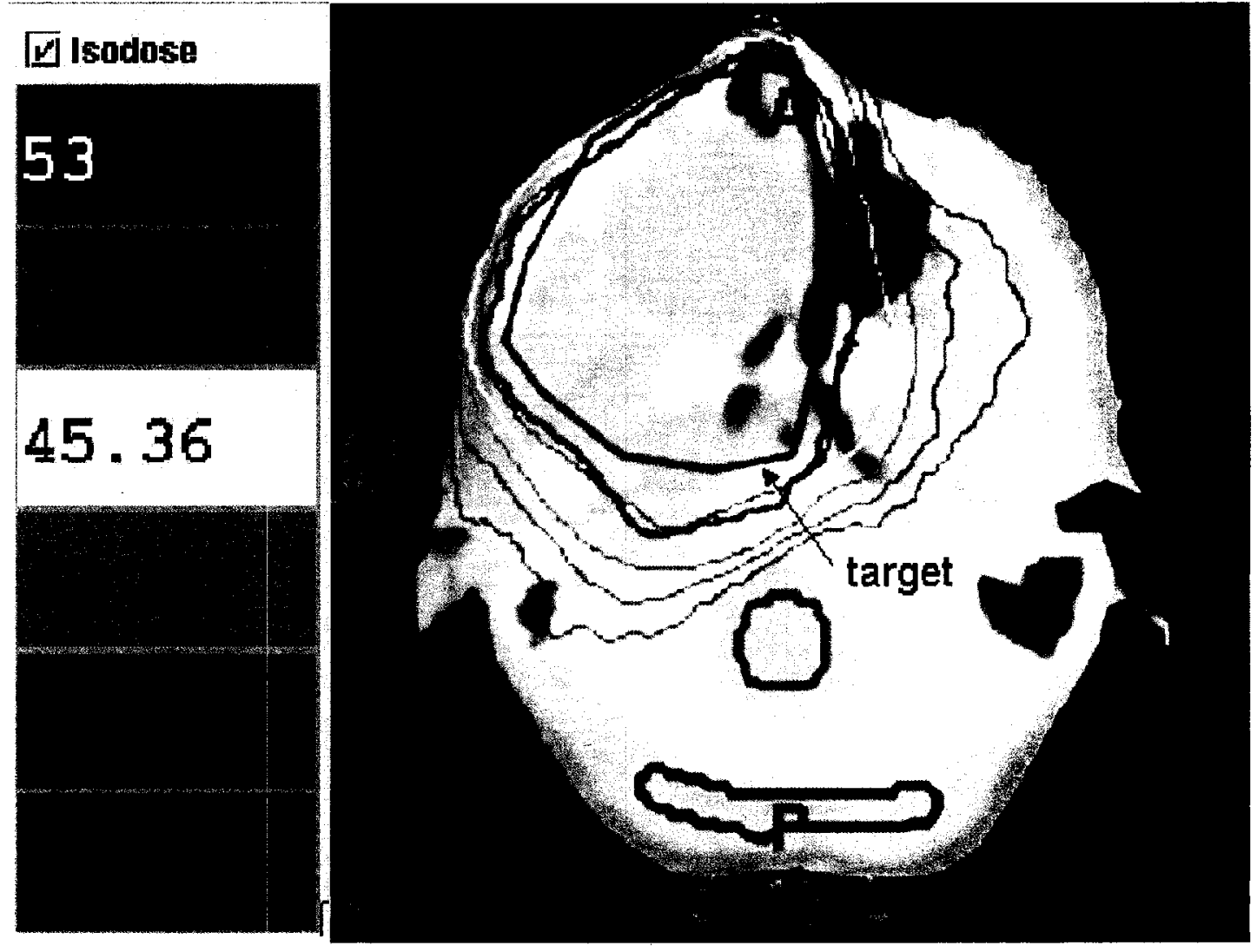

Fig.5.7 Isodose curves generated from the treatment plan for 'Patient $A$ '. The prescribed target volume, located to the right of the nose, is shown as well as areas of dose that follow the legend on the left. The red curve, for example, outlines a region that receives at least $50.4 \%$ of the prescribed dose. 


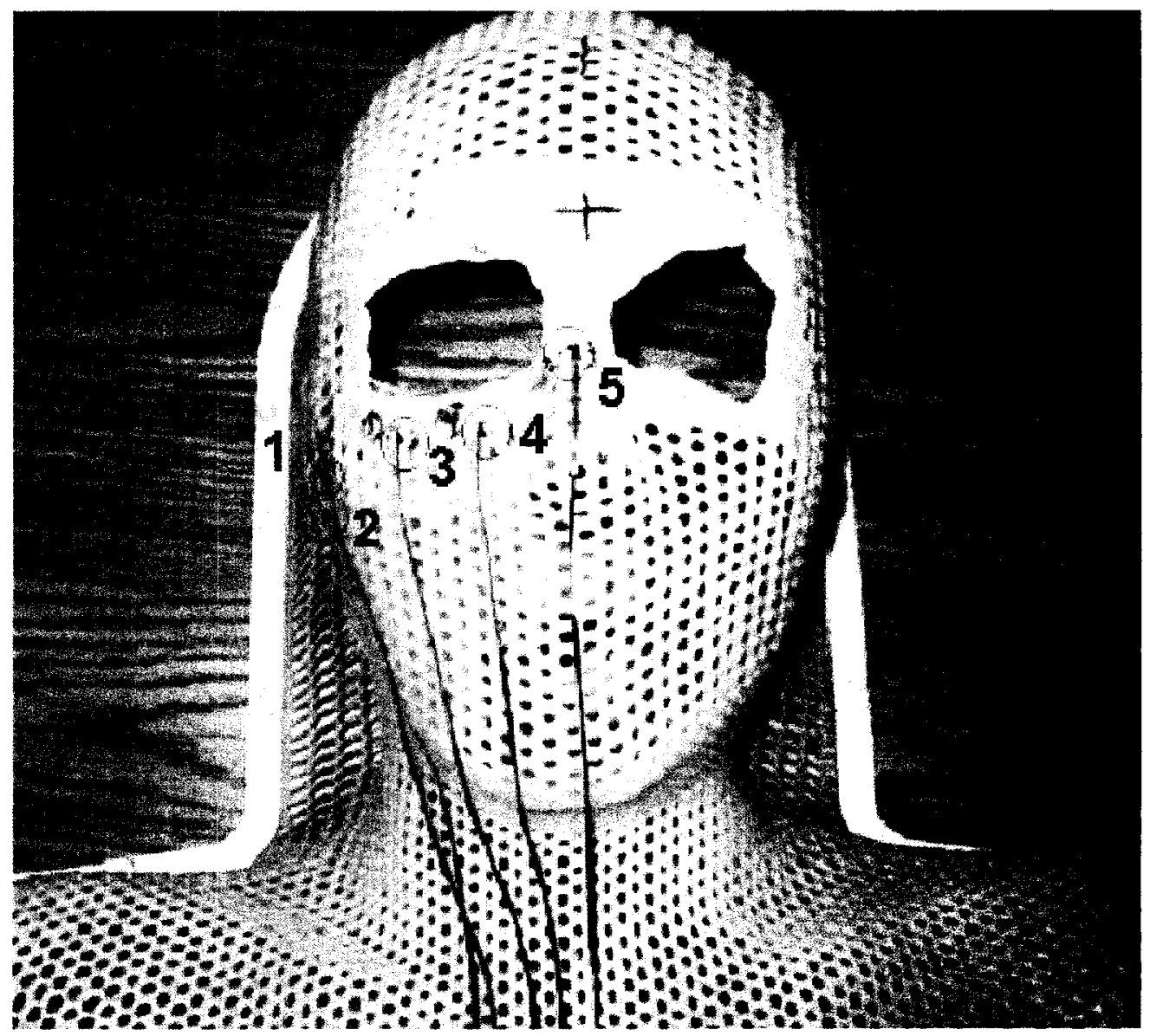

Fig.5.8 The immobilization mask for 'Patient A' with MOSFETs attached to the five points of interest. 
Each day, care was taken to assure accurate positioning of the MOSFETs on the mask, and any misplacement that happened during the treatment delivery was noted. MOSFET measurements were taken during ten treatments for each patient and measurements that were compromised because of errors such as misplacement and twisting of the detector from bubble-down to bubble-up position were not included in the analysis. TLDs were prepared according to the manufacturers guidelines and dose measurements were taken at the same reference points for comparison with the MOSFET readings.

During the original CT scan, some patients had ball bearings, bb's, placed on the immobilization mask that were visible on the CT images, defining the reference points for the dose calculations. These points were used to get the calculated treatment plan dose at each reference point for comparison with measured values. For measurements on two of the patients, the MOSFETs used had two radio-opaque markers, one on either side of the sensitive volume. These markers could be seen on the daily scan done on the Tomotherapy unit, and the location of the MOSFET detector could be interpolated. Once the therapists had made adjustments to the patient's position, a calculated dose value was obtained for the points at which the MOSFET detector was located in the scan. This method allowed us to track the daily treatment plan dose as well as to evaluate the positioning of the MOSFETs for these trials. 


\subsubsection{Results}

A total of $\mathbf{2 1 0}$ measurements were taken on five different head and neck patients. All high sensitivity MOSFETs were used on three of the patients, and all standard sensitivity MOSFETs were used on the other two. An example of a set of measurements made on one of the patients is shown in Table 5.4. In total, eleven measurements were excluded from calculations due to misplacement of the detector during treatment. Such errors were avoided later in the experiment as the methods used to attach the MOSFETs to the immobilization mask were improved. The time needed each day to set-up for the in vivo measurements did not increase the overall treatment time, making the use of MOSFETs convenient.

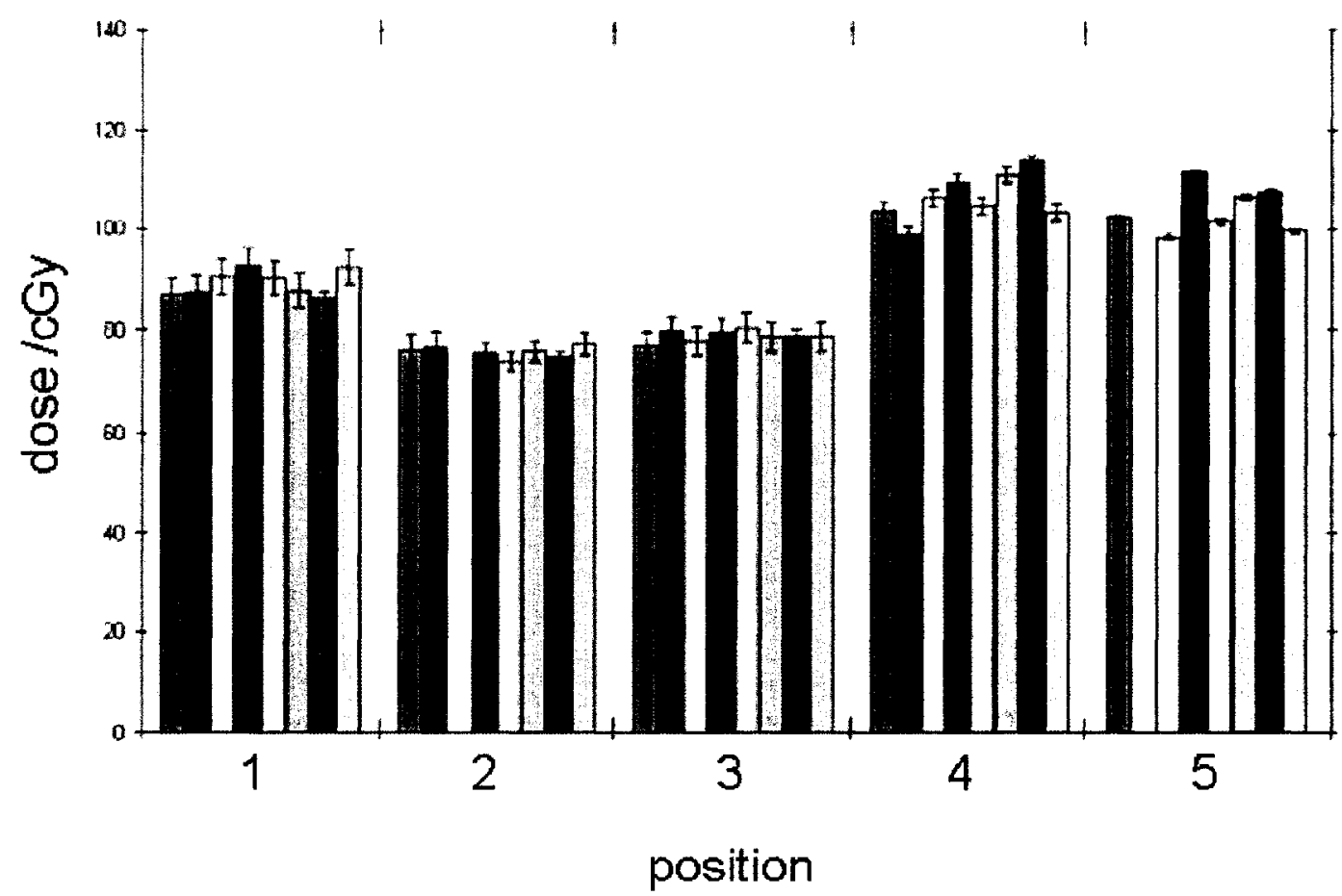

Fig.5.9 MOSFET dose measurements taken on 'Patient E' at five points of interest. Measurements were taken on eight different days, and readings that were compromised due to movement of the detector during treatment have been omitted. The uncertainty shown is from the standard deviation of the original calibration factors. 
Table 5.4 In vivo dose measurements and Tomotherapy treatment plan dose values for 'Patient E' treated on Tomotherapy

\begin{tabular}{|c|c|c|c|c|c|}
\hline \multirow{2}{*}{ Position } & \multirow{2}{*}{$\begin{array}{c}\text { MOSFET Dose } \\
\text { (CGy) }\end{array}$} & \multicolumn{2}{|c|}{ TLD } & \multicolumn{2}{|c|}{ Treatment Plan } \\
\cline { 3 - 6 } & Dose (CGy) & $\begin{array}{c}\text { \% Diff from } \\
\text { MOSFET }\end{array}$ & Dose (CGy) & $\begin{array}{c}\text { \% Diff from } \\
\text { MOSFET }\end{array}$ \\
\hline 1 & $88.5 \pm 3.6 \%$ & $90.0 \pm 9.3 \%$ & $1.6 \%$ & $99.7 \pm 6.1 \%$ & $10.2 \%$ \\
\hline 2 & $74.8 \pm 2.7 \%$ & $75.3 \pm 6.7 \%$ & $0.7 \%$ & $77.7 \pm 1.1 \%$ & $3.1 \%$ \\
\hline 3 & $79.1 \pm 1.4 \%$ & $79.9 \pm 7.9 \%$ & $1.0 \%$ & $106.0 \pm 4.1 \%$ & $28.1 \%$ \\
\hline 4 & $106.4 \pm 4.5 \%$ & $108.8 \pm 2.5 \%$ & $2.2 \%$ & $84.0 \pm 5.8 \%$ & $-25.7 \%$ \\
\hline 5 & $103.7 \pm 4.1 \%$ & $110.4 \pm 12.4 \%$ & $6.2 \%$ & $99.6 \pm 6.1 \%$ & $-10.2 \%$ \\
\hline
\end{tabular}


The inter-fraction deviation of the MOSFET readings at each point ranged from $1.4 \%$ to $6.6 \%$. The overall average agreement with the TLD measurements was $(3.26 \pm 0.03) \%$. The agreement with the treatment plan doses was similar to the results found with the RANDO phantom. The average absolute difference between the MOSFET measured dose and the calculated treatment plan dose was $(12.2 \pm 7.5) \%$. The calculated treatment plan doses varied from overestimating to underestimating the measured dose depending on the point of interest. These results confirmed the inconsistencies in the calculation of surface doses by the treatment planning system. There are a number of reasons that could cause such problems, including the partial volume effect, which accounts for the finite size of the voxels. Each voxel is assigned a density, measured in Hounsfield units, which is used for the dose calculation. Since there is only one density value assigned to each voxel, problems can arise at interfaces between two different materials where there can be more than one material in a voxel, leading to inaccurate dose estimation. This effect is particularly significant near the air-surface interface, where the densities of the two materials are so different. Treatment plans for head and neck patients are done on the finest grid size possible to minimize this problem; however it is obvious that surface dose calculations are still affected. To investigate this problem further, a cylindrical solid water phantom was scanned using KVCT and the images were sent to the Tomotherapy treatment planning system. The phantom had a smaller cylindrical 'lung' insert with a density of $0.40 \mathrm{~g} / \mathrm{cm}^{3}$. Profiles of the Hounsfield units assigned to the voxels were then recorded using the Tomotherapy software. As seen in 
Fig.5.10, the boundary between any two materials is not sharp, but is instead stretched over a region encompassing both materials. Since the densities in this interface region are from a combination of the true densities of the two materials, the points of interest used in this experiment, which were at the air-surface interface, would have been significantly affected.

There are several other problems with surface dose calculations including incomplete electron transfer modeling and the use of beams that enter tangentially to the skin. As discussed earlier, head and neck cancer treatment plans often require a high dose close to the surface. The build-up region of beams that enter perpendicular to the surface force the maximum dose to occur deeper in the tissue. In some cases, the use of bolus is sufficient to achieve the required dose close enough to the surface, however in other situations the treatment plan can be optimized by having beams enter tangentially to the skin. As the beam travels on an angle through the tissue, the build-up region can occur before reaching the target. Dose delivery using beams entering this way is particularly sensitive to small positioning errors and also the treatment planning system has trouble modeling such beams. All of these factors can lead to the inaccuracies identified using our in vivo measurements. 


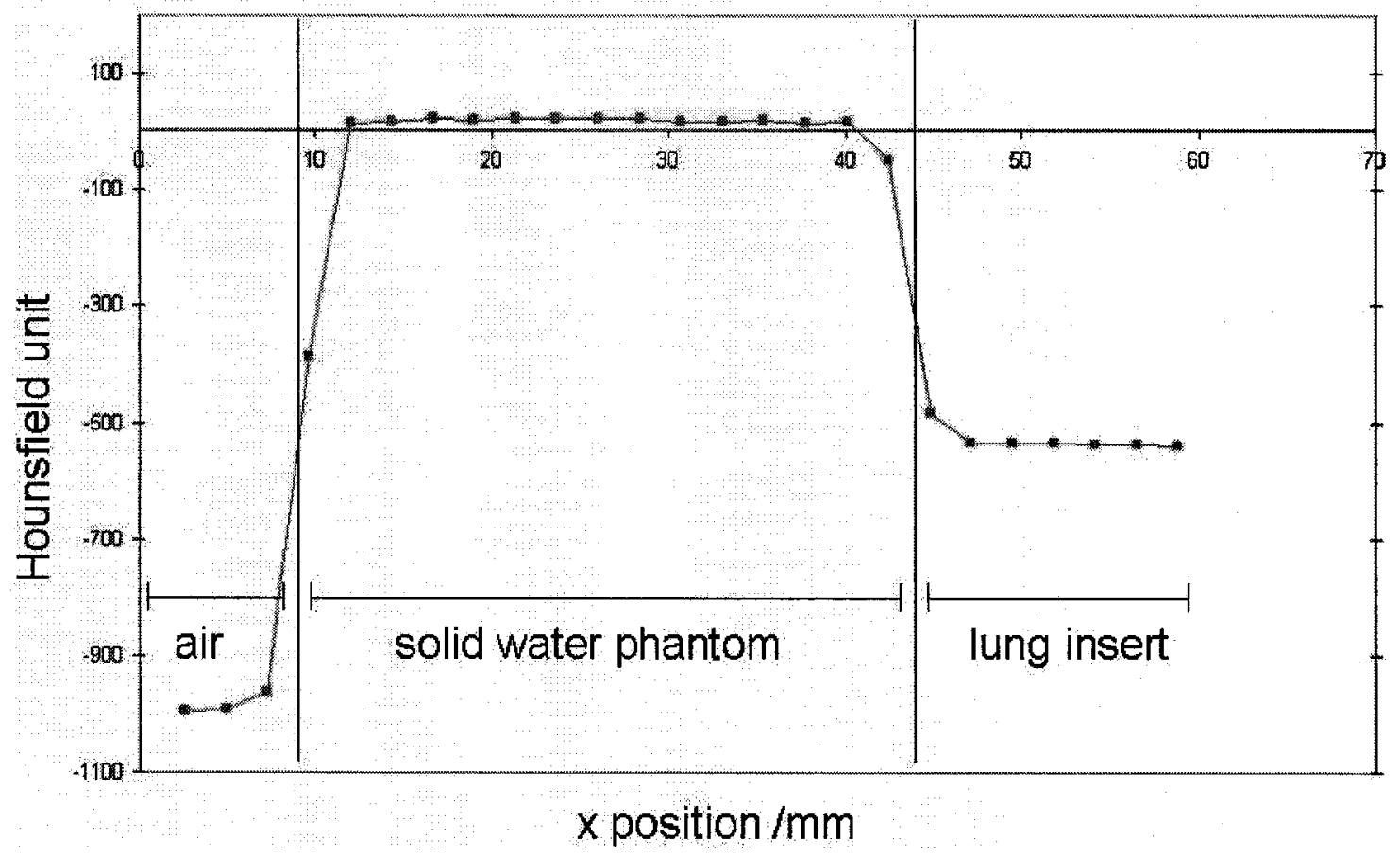

Fig.5.10 A profile of Hounsfield units from a kVCT of cylindrical solid water phantom with a lung insert, as assigned by the Tomotherapy treatment planning software. 
a.

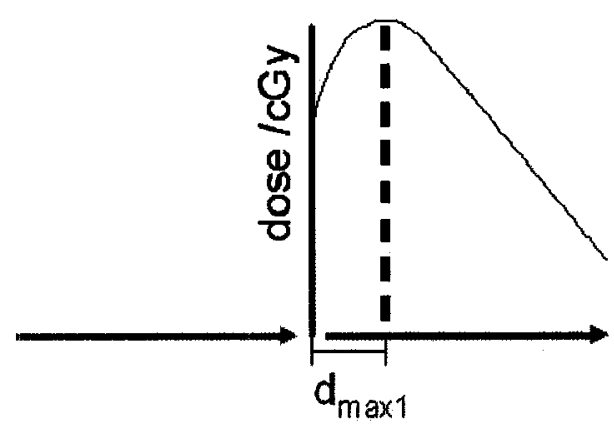

Air

Tissue b.

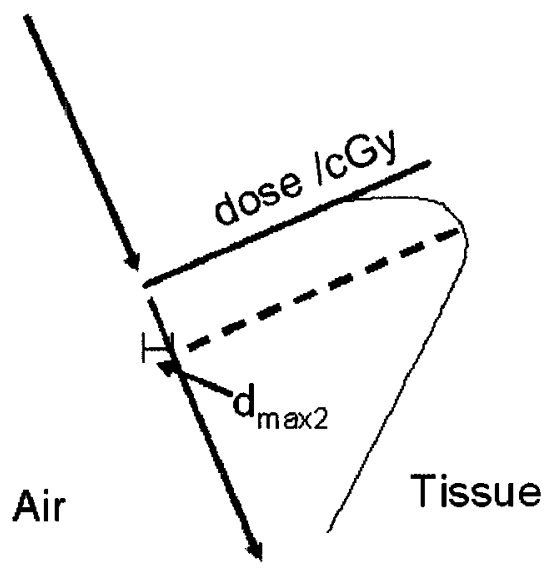

Fig.5.11 A dose profile of a beam entering tissue a. perpendicular to the surface and $b$. on an angle. The depth of maximum dose, $d_{\max }$, can be brought closer to the surface by using beams entering on angle, as seen with $d_{\max 2}$, a method commonly used in head and neck treatment plans. 


\section{Chapter 6}

\section{Conclusions}

The average calibration factor found for the MOSFETs tested in this study was $0.345 \pm 2.5 \% \mathrm{cGy} / \mathrm{mV}$ for the high sensitivity MOSFETs tested and $0.901 \pm 2.4 \%$ cGy/mV for the standard sensitivity MOSFETs. The MOSFET measured surface doses had an average agreement with ion chamber measurements of $1.55 \%$ for the high sensitivity MOSFET and $5.23 \%$ for the standard sensitivity MOSFET when averaged over all trials and field sizes tested. No significant dependence of the calibration factor on field size or on accumulated dose was found for the MOSFETs.

The MOSFETs performed well when used in the Tomotherapy Hi-Art unit and did not increase the overall treatment set-up time when used for patient measurements. The MOSFET measurements on the head and neck patients treated with Tomotherapy had an average agreement of $(3.26 \pm 0.03) \%$ with TLD measurements, however the absolute average of the difference between the MOSFET measurements and the treatment plan skin doses was $(12.2 \pm 7.5) \%$. This further demonstrated the need for in vivo dosimetry during such treatments as the treatment planning system does not always provide reliable dose estimations in the surface and build-up regions. The MOSFET measured patient skin doses had good reproducibility, with inter-fraction deviations ranging from $1.4 \%$ to $6.6 \%$ and similar results were found from trials using a RANDO phantom. 
In conclusion, it was found that MOSFETs are suitable detectors for surface dose measurements in both conventional beam and Tomotherapy treatments and they can provide valuable skin dose information in areas where the treatment planning system may not be accurate. 


\section{REFERENCES}

[1] Halnan K. One hundred years of radiotherapy in Britain. In:Thomas AMK, Isherwood I, Wells PNT, editors. The invisible light. Oxford: Blackwell Science Ltd, $1995 ; 44-55$

[2] Intensity Modulated Radiation Therapy Collaborative Working Group. Intensity-modulated Radiotherapy: current status and issues of interest. Int J Radiat Oncol Biol Phys. 2001; 51:880-914

[3] Mackie TR, Olivera GH, Kapatoes JM, et al. Helical tomotherapy. In: Palta P, Mackie TR, editors. Intensity-modulated radiation therapy: the state of the art. College Park MD: American Association of Physicists in Medicine, 2003:247-284 [4] Thomas SJ and Hoole ACF. The effect of optimization on surface dose in intensity modulated radiotherapy (IMRT). Phys Med Biol. 2004; 49: 4919-4928 [5] Lee N, Chuang C, Quivey JM, et al. Skin toxicity due to intensity-modulated radiotherapy for head-and-neck carcinoma. Int J Radiat Oncol Biol Phys. 2002; 53:630-7

[6] Chung H., Jin H, Dempsey JF et al. Evaluation of surface and build-up region dose for intensity-modulated radiation therapy in head and neck cancer. Med Phys. 2005; 32:2682-2689

[7] Chuang CF, Verhey LJ, and Xia P. Investigation of the use of MOSFET for clinical IMRT dosimetric verification. Med Phys. 2002; 29:1109-1115.

[8] Marcie S, Charpiot E, Bensadoun R-J. In vivo measurements with MOSFET detectors in Oropharynx and Nasopharynx intensity-modulated radiation therapy. Int J Radiat Onc Biol Phys. 2005; 61:1603-1606 
[9] Lanson JH, Essers M, Meijer GJ, et al. In vivo dosimetry during conformal radiotherapy: Requirements for and findings of a routine procedure. Radiother Oncol. 1999; 52:51-59

[10] Soubra M, Cygler J, Mackay G. Evaluation of dual bias dual metal oxidesilicon semiconductor field effect transistor detector as radiation dosimeter. Med Phys. 1994; 21: 567-572

[11] Scalchi P, Francescon P, Rajaguru P. Characterization of a new MOSFET detector configuration for in vivo skin dosimetry. Med Phys. 2005; 32:1571-1578 [12] Scarantino CW, Ruslander DM, Nagle HT, et al. An implantable radiation dosimeter for use in external beam radation therapy. Med Phys. 2004; 31:26582671

[13] Scarantino CW, Rini CJ, Aquino MA, et al. Initial clinical results of an in vivo dosimeter during external beam radiation therapy. Int J Radiat Onc Biol Phys. $2005 ; 62: 606-613$

[14] Ramaseshan R, Kohli KS, Zhang TJ, et al. Performance characteristics of a microMOSFET as an in vivo dosimeter in radiation therapy. Phys Med Biol. $2004 ; 49,4031-4048$

[15] Ramaseshan R, Russell S, O'Brien P. Clinical dosimetry using MOSFETs. Int J Radiat Onc Biol Phys. 1997; 37:959-964 [16] Jornet N, Carrasco P, Jurado D, et al. Comparison study of MOSFET detectors and diodes for entrance in vivo dosimetry in $18 \mathrm{MV}$ x-ray beams. Med Phys. 2004; 31:2534-2542 
[17] Essers $\mathrm{M}$ and Mijnheer BJ. In vivo dosimetry during external photon beam radiotherapy. Int J Radiat Onc Biol Phys. 1999; 43: 245-259

[18] Kron T, Buston M, Wong $T$, et al. Readout of thermoluminescence dosimetry chips using a contact planchet heater. Austras Phys Eng Sci Med. 1993:16:137142

[19] Kron T, Butsnob M, Huntb F, et al. TLD extrapolation for skin dose determination in vivo. Radiother Oncol 1996; 41: 119-123 [20] Loncol T, Greffe LJ, Vynckier S, et al. Entrance and exit dose measurements with semiconductors and thermoluminescent dosemeters: a comparison of methods and in vivo results. Radiother Oncol 1996; 41:179-187 [21] Nutting C, Dearnaley DP, Webb S. Intensity modulated radiation therapy: a clinical review. BJR. 2000; 73: 459-469

[22] Aznar M, Andersen C, Bøtter-Jensen L, et al. Real-time optical-fibre luminescence dosimetry for radiotherapy: physical characteristics and applications in photon beams. Phys Med Biol. 2004; 49:1655-1669 [23] Webb S. The physical basis of IMRT and inverse planning. BJR 2003; 76:678-89

[24] Carol M. Peacock: a system for planning and rotational delivery of intensitymodulated fields. Int J Imag Syst Technol 1995; 6:56-61

[25] Forrest LJ, Mackie TR, Ruchala K, et al.The utility of megavoltage computed tomography images from a helical tomotherapy system for setup verification purposes. Int J Rad Onc Biol Phys. 2004; 60: 1639-1644 
[26] Mackie TR, Holmes TW, Swerdloff S, et al. Tomotherapy: a new concept in the delivery of dynamic conformal radiotherapy. Med Phys. 1993; 20: 1709-1719 [27] Scalchi P and Francescon P. Calibration of a MOSFET detection system for 6-MV in vivo dosimetry. Int J Radiat Onc Biol Phys.1998; 40: 987-993

[28] Cygler J, Saoudi A, Perry G, et al. Feasibility study of using MOSFETs for in vivo dosimetry during permanent low-dose-rate prostate implants. Rad Onc 2006; 80:296-301

[29] Cheung T, Butson MJ, Yu PKN. Effects of temperature variation on MOSFET dosimetry. Phys Med Biol. 2004; 49:191-196

[30] Butson MJ, Rozenfeld A, Mathus JN, et al. A new radiotherapy surface dose detector: The MOSFET. Med Phys. 1996; 23: 655-658

[31] Rikner G and Grusell E. Effect of radiation damage on p-type silicon detectors. Phys Med Biol. 1983; 28:1261-1267

[32] Rikner G and Grusell E. General specification for silicon semi-conductors for use in radiation dosimetry. Phys Med Biol. 1987; 32:1109-1117 [33] Wood JJ and Mayles WP. Factors affecting the precision of TLD dose measurements using an automatic TLD reader. Phys Med Biol. 1995; 40:309-313 [34]McGhee PL, Humphreys S, Dunscombe, P. An efficient approach to routine TL dosimetry. Med Dosim. 1993; 18:187-191 [35] Fairbanks EJ and DeWerd, LA. Thermoluminescent characteristics of LiF: Mg, Ti from three manufacturers. Med Phys. 1993; 20:729-731 [36] Higgins PD, Alaei P, Gerbi BJ, et al. In vivo diode dosimetry for routine quality assurance in IMRT. Med Phys. 2003; 30: 3118-3123 
[37] Lee PC, Sawicka JM, Glasgow GP. Patient dosimetry quality assurance program with a commercial diode system. Int J Radiat Oncol Biol Phys. 1994; 29:1175-1182

[38] Colussi VC, Beddar AS, Kinsella TJ, et al. In vivo dosimetry using a single diode for megavoltage photon beam radiotherapy: Implementation and response characterization. J Appl Clin Med Phys. 2001; 2:210-218 [39] Howlett S, Duggan L, Bazley S, et al. Selective in vivo dosimetry in radiotherapy using p-type semiconductor diodes: A reliable quality assurance procedure. Med Dosim. 1999; 24:53-56

[40] Alecu R, Loomis T, Alecu J, et al. Guidelines on the implementation of diode in vivo dosimetry programs for photon and electron external beam therapy. Med Dosim. 1999; 24:5-12

[41] Saini AS and Zhu TC. Temperature dependence of commercially available diode detectors. Med Phys. 2002; 29:622-630 [42] Jursinic PA. Implementation of an in vivo diode dosimetry program and changes in diode characteristics over a four-year clinical history. Med Phys. $2001 ; 28: 1718-1726$

[43] Fontenla DP, Yaparpalvi R, Chui CS, et al. The use of diode dosimetry in quality improvement of patient care in radiation therapy. Med Dosim. 1996; $21: 235-241$

[44] Butson MJ, Yu PKN, Metcalfe PE. Extrapolated surface dose measurements with radiochromic film. Med Phys. 1999; 26: 485-488 
[45] Podgorsak EB et al. Radiation oncology physics: a handbook for teachers and students. Vienna: International Atomic Energy Agency, 2005.

[46] Leunens G, Van Dam J, Dutreix A, et al. Quality assurance in radiotherapy by in vivo dosimetry. Entrance dose measurements, a reliable procedure. Radiother Oncol. 1990; 17:141-151

[47] TG-51 AAPM, AAPM's TG-51 protocol for clinical reference dosimetry of high-energy photon and electron beams. Med Phys. 1999; 26:1847-1870 [48] Rogers DWO. "Fundamentals of dosimetry based on absorbed dose standards" in Teletherapy: Present and Future, edited by Mackie T. R. and Palta J. R. AAPM Advanced Medical Publishing, Madison, 1996; 319-356 [49] Leunens G, Verstraete J, Dutreix A, et al. Assessment of dose inhomogeneity at target level by in vivo dosimetry: Can the recommended $5 \%$ accuracy in the dose delivered to the target volume be fulfilled in daily practice? Radiother Oncol. 1992; 25:242-250 [50] Mayles WPN, Heisig S, Mayles HMO. Treatment Verification and in vivo Dosimetry. Radiotherapy Physics by J. R. Williams and D. I. Thwaites. Oxford: Oxford University Press, 1993:227-251

[51] Millwater CJ, MacLeod AS, Thwaites DI. In vivo semiconductor dosimetry as part of routine quality assurance. BJR. 1998; 71:661-668 [52] Voordeckers M, Goossens H, Rutten J, et al. The implementation of in vivo dosimetry in a small radiotherapy department. Radiother Oncol. 1998; 47:45-48 [53] LoSasso TJ. "Quality Assurance of IMRT" in A Practical Guide to Intensity 
Modulated Radiation Therapy. Madison, WI: Medical Physics Publishing, 2003:147-167

[54]. Ezzell A, Galvin JM, Low D, et al. Guidance document on delivery, treatment planning, and clinical implementation of IMRT: Report of the IMRT subcommittee of the AAPM radiation therapy committee. Med Phys. 2003; 30: 2089-2115

[55] Mutic S and Low DA. Superficial doses from serial tomotherapy delivery. Med Phys. 2001; 27; 163-165

[56] Mackie TR, Balog J, Ruchala K, et al. Tomotherapy. Semin Radiat Oncol. $1999 ; 9: 108-117$

[57] Fenwick JD, Tome WA, Jaradat HA, et al. Quality assurance of a helical tomotherapy machine. Phys Med Biol. 2004; 49: 2933-2953

[58] Mohan R, Wang X, Jackson A et al., The potential and limitations of the inverse radiotherapy technique. Radiother Oncol. 1994; 32: 232-248 [59] Low DA and Mutic S. A commercial IMRT treatment planning dose calculation algorithm. Int J Radiat Oncol Biol Phys 1988; 41:933-937 [60] Van Dyk J, Barnett RB, Cygler JE, et al. Commissioning and quality assurance of treatment planning computers. Med Phys 1993; 26: 261-273 [61] Verellen D, Linthout N, Berge DVD, et al. Initial experience with intensitymodulated conformal radiation therapy for treatment of the head and neck region. Int J Radiat Oncol Biol Phys 1997; 39: 99-114 [62] Low DA, Chao KC, Gerber RL, et al. Quality assurance of arc-based IMRT patient treatments: Preliminary results. Int J Radiat Oncol Biol Phys 1997; 39:149 
[63] Jeraj R, Mackie TR, Balog J, et al. Radiation characteristics of helical Tomotherapy. Med Phys. 2004; 31: 396-404

[64] Hounsell R and Wilkinson JM. Electron contamination and build-up doses in conformal radiotherapy fields. Phys Med Biol. 1999; 44:43-55

[65] Quach KY, Morales J, Butson MJ, et al. Measurement of radiotherapy x-ray skin dose on a chest wall phantom. Med Phys. 2000; 27: 1676-1680 [66] Dogan N and Glasgow GP. Surface and build-up region dosimetry for obliquely incident intensity modulated radiotherapy $6 \mathrm{MV} x$ rays. Med Phys. 2003; 30:3091-3096

[67] Kim S, Akpati HC, Kielbasa JE, et al. Evaluation of intrafraction patient movement for CNS and head \& neck IMRT. Med Phys. 2004; 31:500-506 [68] Kim S, Akpati HC, Li JG, et al. An immobilization system for claustrophobic patients in head-and-neck intensity-modulated radiation therapy. Int J Radiat Oncol Biol Phys. 2004; 59:1531-1539

[69] Webb S. The physics of conformal radiotherapy and advances in technology. Bristol: IOP Publishing, 1997.

[70] Fuks Z, Horwich A. Clinical and technical aspects of conformal therapy. Radiother Oncol. 1993; 29: 219-220

[71] Brahme A. Optimisation of stationary and moving beam radiation therapy techniques. Radiother Oncol. 1988; 12:129-140

[72] Boyer AL, Geis P, Grant W, et al. Modulated beam conformal therapy for head and neck tumours. Int J Radiat Oncol Biol Phys. 1997; 39: 227-236 
[73] Goitein M and Niemierko A. Intensity modulated therapy and inhomogeneous dose to the tumour: a note of caution. Int J Radiat Oncol Biol Phys. 1996; 36:519-522

[74] De Neve W, De Gersem W, Derycke S, et al. Clinical delivery of intensity modulated conformal radiotherapy for relapsed or second primary head and neck cancer using a multileaf collimator with dynamic control. Radiother Oncol. 1999; $50: 301-314$

[75] Martel MK, Eisbruch A, Lawrence TS, et al. Spinal cord dose from standard head and neck irradiation: implications for three-dimensional treatment planning. Radiother Oncol. 1997; 47:185-189 